\title{
Enabling fast, stable and accurate peridynamic computations using multi-time-step integration ${ }^{\text {th }}$
}

\author{
P. Lindsay ${ }^{\mathrm{a}}$, M. L. Parks ${ }^{\mathrm{b}, * *}$ A. Prakash ${ }^{\mathrm{a}, *}$ \\ ${ }^{a}$ Lyles School of Civil Engineering, Delon and Elizabeth Hampton Hall of Civil \\ Engineering, 550 Stadium Mall Drive, West Lafayette, IN 47907-2051 \\ ${ }^{b}$ Sandia National Laboratories, P.O. Box 5800, MS 1320, Albuquerque, NM 87185-1320
}

\begin{abstract}
Peridynamics is a nonlocal extension of classical continuum mechanics that is well-suited for solving problems with discontinuities such as cracks. This paper extends the peridynamic formulation to decompose a problem domain into a number of smaller overlapping subdomains and to enable the use of different time steps in different subdomains. This approach allows regions of interest to be isolated and solved at a small time step for increased accuracy while the rest of the problem domain can be solved at a larger time step for greater computational efficiency. Performance of the proposed method in terms of stability, accuracy, and computational cost is examined and several numerical examples are presented to corroborate the findings.
\end{abstract}

Keywords: Peridynamics, Multi-Time-Step, Nonlocal, Fracture

\footnotetext{
This material is based upon work supported by the U.S. Department of Energy Office of Science, Office of Advanced Scientific Computing Research, Computer Science program.

${ }^{*}$ Corresponding author

** Sandia is a multiprogram laboratory operated by Sandia Corporation, a Lockheed Martin Company, for the U.S. Department of Energy under contract DE-AC04-94AL85000.

Email addresses: plindsay@purdue.edu (P. Lindsay), mlparks@sandia.gov (M. L. Parks), aprakas@purdue.edu (A. Prakash)
} 


\section{Introduction}

The classical theory of solid mechanics has been used to model a wide range of physical phenomena. However, there are certain problems for which classical solid mechanics is not well-suited, such as crack initiation and propagation. This is because the partial differential equations used in classical solid mechanics are undefined along discontinuities, and consequently formulations based on classical theory must be adapted to capture discontinuous behavior [1,2]. Existing methods for modeling fracture include the XFEM and GFEM methods $[3,4]$ that are based on partition of unity enrichment methods [5, 6], cohesive zone methods [7], meshfree methods [8, 9, 10], and a number of different approaches that use molecular dynamics. Some authors have also explored nonlocal models to circumvent the limitations posed by classical solid mechanics $[11,12,13,14]$.

Peridynamics (PD) is a nonlocal extension of classical solid mechanics where the basic equations are formulated in terms of integral equations that are well defined along discontinuities (see [15, 16]). However, for large, complex problems involving multiple scales in space and time, the entire PD model must typically be solved at a very small time step, which is often extremely computationally intensive. Two common methods that have been used to address this issue in continuum solid and structural mechanics are domain decomposition (DD) and multi-time-step (MTS) methods (see Prakash and coworkers $[17,18,19])$. DD and MTS methods allow a computational domain to be divided into multiple subdomains that can be solved with different time steps and integration techniques to reduce the computational cost.

This paper formulates a multi-time-step method for peridynamics (MTSPD) and studies its performance in terms of stability, accuracy and computational cost. It is shown that two PD subdomains can be solved independently

using different time steps, that these solutions can be coupled efficiently, and furthermore that the coupled system accurately represents the response ex- 
hibited by the original undecomposed model. To the best of the authors' knowledge, this work represents the first instance where the MTS approach has been formulated for decomposing and coupling nonlocal models such as PD.

\section{Peridynamics Background and Theory}

PD is a nonlocal extension of classical solid mechanics that was originally developed to address some limitations of the classical theory by reformulating the classical partial differential equations as integro-differential equations. Discontinuous displacements do not require special treatment with PD, and thus discontinuities can be easily modeled at realistic length scales. The basic theory of PD was developed by Silling and coworkers (see $[15,16,20])$ and is based on nonlocal interactions between material points that are within each other's neighborhood.

PD is often used in the study of material failure, as seen in an example by Askari et al. [21]. However, PD has also been used to explore many different physical phenomena. Another common application of PD is the study of damage, fracture, and fragmentation resulting from impact (see Silling and Askari [22]). An application to nano-structures has been studied by Bobaru and Silling [23, 24]. Seleson and coworkers [25, 26] have also investigated upscaling molecular dynamics to peridynamics. Gerstle et al. [27] developed a micropolar PD constitutive model for concrete that is able to capture microcracking and damage evolution in concrete structures. These are only a few of the many PD applications to date. A paper by Silling and Lehoucq [20] includes a summary of the literature on PD.

\subsection{Peridynamic formulation}

The basic PD theory is summarized here. The problem domain is represented by a collection of interacting material points as shown in Figure 1, where $\boldsymbol{x}$ represents the position of a point, and $\mathcal{H}_{x}:=\left\{\boldsymbol{x}^{\prime} \in \mathbb{R}^{3}:\left\|\boldsymbol{x}^{\prime}-\boldsymbol{x}\right\| \leq \delta\right\}$ 
is the neighborhood of $\boldsymbol{x}$ containing all points $\boldsymbol{x}^{\prime}$ that $\boldsymbol{x}$ interacts with in the body $\Omega$. In general, $\mathcal{H}_{x}$ can be thought of as a sphere of some radius $\delta$, centered at $\boldsymbol{x}$. The radius $\delta$ is called the horizon.

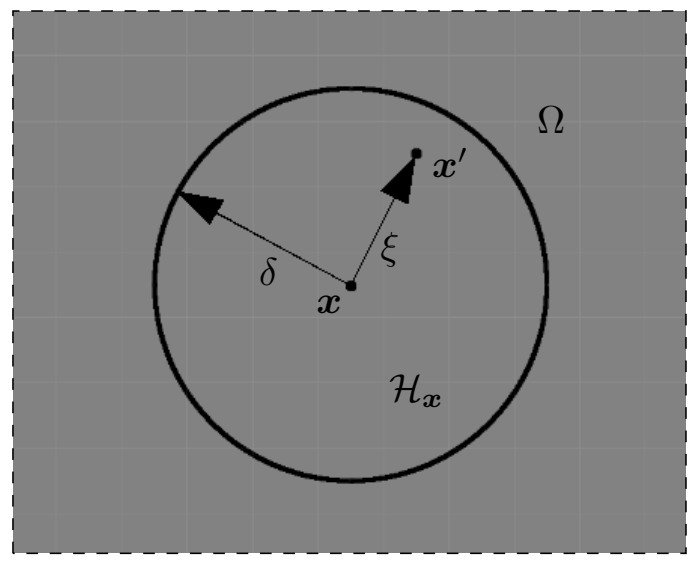

Figure 1: The spherical neighborhood $\mathcal{H}_{\boldsymbol{x}}$ of a material point $\boldsymbol{x}$

The governing integro-differential equation of motion for PD is given by

$$
\rho(\boldsymbol{x}) \ddot{\boldsymbol{u}}(\boldsymbol{x}, t)=\int_{\mathcal{H}_{\boldsymbol{x}}}\left\{\underline{\boldsymbol{T}}[\boldsymbol{x}, t]\left\langle\boldsymbol{x}^{\prime}-\boldsymbol{x}\right\rangle-\underline{\boldsymbol{T}}\left[\boldsymbol{x}^{\prime}, t\right]\left\langle\boldsymbol{x}-\boldsymbol{x}^{\prime}\right\rangle\right\} d V_{\boldsymbol{x}^{\prime}}+\boldsymbol{b}(\boldsymbol{x}, t),
$$

where $\boldsymbol{u}(\boldsymbol{x}, t)$ denotes the displacement field, $\boldsymbol{b}(\boldsymbol{x}, t)$ represents the external body force density field, and $\rho$ is the material density. $\underline{\boldsymbol{T}}$ is the force vector state (a generalization of a second-order tensor) that operates on the bond vector $\boldsymbol{\xi}=\boldsymbol{x}^{\prime}-\boldsymbol{x}$ between $\boldsymbol{x}^{\prime}$ and $\boldsymbol{x}$ and returns the force state vector field $\underline{\boldsymbol{T}}[\boldsymbol{x}, t]\left\langle\boldsymbol{x}^{\prime}-\boldsymbol{x}\right\rangle$.

A particular class of materials, called ordinary materials, leads to a useful simplification of the PD equation of motion. Ordinary materials are those for which the force vector state $\underline{\boldsymbol{T}}$ can be represented as $\underline{\boldsymbol{T}}=\underline{t} \underline{\boldsymbol{M}}$, where $\underline{t}$ is a scalar state and $\underline{M}$ is the deformed direction vector state such that $\underline{\boldsymbol{M}}\left\langle\boldsymbol{x}^{\prime}-\boldsymbol{x}\right\rangle$ yields a unit vector pointing from the deformed position of $\boldsymbol{x}$ to the deformed position of $\boldsymbol{x}^{\prime}$. To simplify the exposition for the remainder of 
the discussion, the following relation on the scalar state $\underline{t}$ is assumed,

$$
\underline{t}[\boldsymbol{x}, t]\left\langle\boldsymbol{x}^{\prime}-\boldsymbol{x}\right\rangle=1 / 2 f\left(\boldsymbol{u}\left(\boldsymbol{x}^{\prime}, t\right)-\boldsymbol{u}(\boldsymbol{x}, t), \boldsymbol{x}^{\prime}-\boldsymbol{x}\right),
$$

where $f$ is a scalar function. Ordinary materials satisfying (2) are referred to as "bond-based" PD materials. Using the above relationships, the equation of motion (1) can be reformulated as

$$
\rho(\boldsymbol{x}) \ddot{\boldsymbol{u}}(\boldsymbol{x}, t)=\int_{\mathcal{H}_{\boldsymbol{x}}} \boldsymbol{f}\left(\boldsymbol{u}\left(\boldsymbol{x}^{\prime}, t\right)-\boldsymbol{u}(\boldsymbol{x}, t), \boldsymbol{x}^{\prime}-\boldsymbol{x}\right) d V_{\boldsymbol{x}^{\prime}}+\boldsymbol{b}(\boldsymbol{x}, t),
$$

where $\boldsymbol{f}$ is a pairwise force function, representing the force per volume squared that point $\boldsymbol{x}^{\prime}$ exerts on point $\boldsymbol{x}$ (see $\S 8$ of Silling et al. [16] for details). This equation of motion governs the response of every point $\boldsymbol{x}$ in the problem domain.

The application of boundary conditions is another aspect of peridynamics that differs significantly from classical approaches. For instance, traction boundary conditions are applied as a body force density $\boldsymbol{b}$ along a layer of the boundary. For bond-based peridynamics, this layer must have a width at least equal to the horizon $\delta$.

\subsection{Discretization}

To facilitate computations using (3), a simple discretization that relies on a uniform mesh of cells can be used. A cell $i$ is defined as the set of points $\mathcal{C}^{i}=\left\{\boldsymbol{x}:\left(\boldsymbol{x}-\boldsymbol{x}^{i}\right) \cdot \boldsymbol{e}_{j}<\Delta x / 2 \forall j=1,2,3\right\}$ where $\boldsymbol{e}_{j}$ are basis vectors and $\Delta x$ denotes the spatial extent of the cell in each dimension. With this discretization, the equation of motion is enforced for each cell rather than all material points in the domain. Following the linearized PMB model of Silling and Askari (equation (14) in [28]), we assume a linear bond-based peridynamic model with pairwise force function $\boldsymbol{f}$ given by

$$
\boldsymbol{f}=\boldsymbol{C}\left(\boldsymbol{x}^{\prime}-\boldsymbol{x}\right)\left(\boldsymbol{u}\left(\boldsymbol{x}^{\prime}, t\right)-\boldsymbol{u}(\boldsymbol{x}, t)\right)
$$


where $\boldsymbol{C}\left(\boldsymbol{x}^{\prime}-\boldsymbol{x}\right)$ is the micromodulus function of the material as described in $[15,29]$. Specifically, for this work, the micromodulus is defined using the following scalar valued function:

$$
C\left(\boldsymbol{x}^{\prime}-\boldsymbol{x}\right)=\left(18 K / \pi \delta^{4}\right) /\left\|\boldsymbol{x}^{\prime}-\boldsymbol{x}\right\|
$$

where $K$ is the bulk modulus of the material. For further details on the derivation of the micromodulus, the reader is referred to [15].

For the remainder of the discussion, a uniform material density $\rho$ is assumed, and discretized variables are defined as $\boldsymbol{a}_{n}^{i}:=\ddot{\boldsymbol{u}}\left(\boldsymbol{x}^{i}, t_{n}\right), \boldsymbol{v}_{n}^{i}:=\dot{\boldsymbol{u}}\left(\boldsymbol{x}^{i}, t_{n}\right)$, $\boldsymbol{d}_{n}^{i}:=\boldsymbol{u}\left(\boldsymbol{x}^{i}, t_{n}\right) \mathrm{c}$, and $\boldsymbol{b}_{n}^{i}:=\boldsymbol{b}\left(\boldsymbol{x}^{i}, t_{n}\right)$. Thus, the equation of motion can be stated in a discretized form for every cell $i$, at any instant of time $t_{n+1}$, as

$$
\rho \boldsymbol{a}_{n+1}^{i}=\sum_{\boldsymbol{x}^{p} \in \mathcal{H}_{\boldsymbol{x}^{i}}} \boldsymbol{C}\left(\boldsymbol{x}^{p}-\boldsymbol{x}^{i}\right)\left(\boldsymbol{d}_{n+1}^{p}-\boldsymbol{d}_{n+1}^{i}\right) V^{p}+\boldsymbol{b}_{n+1}^{i},
$$

where $V^{p}$ is the volume of a cell $p \neq i$ in the neighborhood $\mathcal{H}_{\boldsymbol{x}^{i}}$ of cell $i$. Special care is needed for cells $p$ that only partially intersect the PD neighborhood of cell $i$, i.e. cells $\mathcal{C}^{p} \not \subset \mathcal{H}_{\boldsymbol{x}^{i}}$ such that $\left\|\boldsymbol{x}^{p}-\boldsymbol{x}^{i}\right\| \leq \delta$. In such cases, $V^{p}$ is taken to be only the partial volume that actually intersects the horizon of cell $i$, which is calculated via

$$
\left(\frac{\left|\mathcal{V}^{p} \cap \mathcal{H}_{\boldsymbol{x}^{p}}\right|}{\left|\mathcal{V}^{p}\right|}\right) V^{p},
$$

where $\mathcal{V}^{p}$ represents the set of the vertices of cell $\mathcal{C}^{p}$. A summary of other existing partial volume methods is given in [30].

\subsection{Bond-based damage model}

In this work, in addition to the definitions above, a simple damage model is used to represent inelastic material behavior. As the material deforms under loading, damage is computed by comparing the stretch $s(t)$, given by

$$
s(t)=\frac{\left\|\boldsymbol{y}\left(\boldsymbol{x}^{\prime}, t\right)-\boldsymbol{y}(\boldsymbol{x}, t)\right\|-\left\|\boldsymbol{x}^{\prime}-\boldsymbol{x}\right\|}{\left\|\boldsymbol{x}^{\prime}-\boldsymbol{x}\right\|}, \boldsymbol{y}(\boldsymbol{x}, t)=\boldsymbol{x}+\boldsymbol{u}(\boldsymbol{x}, t)
$$


to some critical stretch $s_{0}$. Given a fracture energy $G_{0}$, the critical stretch can be calculated as:

$$
s_{0}=\sqrt{5 G_{0} /(9 K \delta)}=\sqrt{5 G_{0} /(6 E \delta)}
$$

where $K$ is the bulk modulus and $E$ is the Young's modulus of the material (see $[28])$.

Cracks can be inserted into a material by removing all bonds that cross the cracked location. For the purposes of this paper, we consider a failure model appropriate for brittle materials. If any bond stretches beyond the critical stretch, that bond is assumed to be broken for the remainder of the simulation.

\subsection{Time integration}

When solving discrete PD systems, a time-step scheme must be used to advance the solution through time. In this study, the Newmark- $\beta$ scheme [31] is used. The Newmark- $\beta$ equations are given by

$$
\begin{aligned}
& \boldsymbol{v}_{n+1}^{i}=\boldsymbol{v}_{n}^{i}+\Delta t\left[(1-\gamma) \boldsymbol{a}_{n}^{i}+\gamma \boldsymbol{a}_{n+1}^{i}\right] \\
& \boldsymbol{d}_{n+1}^{i}=\boldsymbol{d}_{n}^{i}+\Delta t \boldsymbol{v}_{n}^{i}+\Delta t^{2}\left[(1 / 2-\beta) \boldsymbol{a}_{n}^{i}+\beta \boldsymbol{a}_{n+1}^{i}\right],
\end{aligned}
$$

where $\boldsymbol{d}_{n+1}^{i}, \boldsymbol{v}_{n+1}^{i}$, and $\boldsymbol{a}_{n+1}^{i}$ denote the discrete approximations to displacement, velocity and acceleration of cell $i$ at time $t_{n+1}$ respectively. The time step is denoted by $\Delta t$ and the parameters $\gamma$ and $\beta$ can be chosen to obtain different time integration schemes. Two common choices for these parameters are i) explicit integration with $\gamma=\frac{1}{2}$ and $\beta=0$ (Velocity Verlet Method, see [32]), and ii) implicit integration with $\gamma=\frac{1}{2}$ and $\beta=\frac{1}{4}$ (Average Acceleration Method). While the Velocity Verlet scheme is computationally very efficient, it is only conditionally stable for time steps smaller than a critical value. On the other hand, despite the higher computational cost of the Average Acceleration method, it is unconditionally stable and the choice of time 
step in this case is primarily governed by accuracy requirements. The choice of time step and integration scheme is usually made by carefully balancing the trade-off between stability and accuracy on one hand and computational cost on the other.

Depending upon the discretization of the domain and the value of the horizon, PD can be very computationally intensive and time consuming. In following sections, techniques for reducing the computational cost associated with large problems in PD are discussed.

\section{Domain Decomposition and Multi-Time-Step Methods}

Large-scale problems in solid and structural mechanics frequently test the limits of computational resources. That in turn places constraints on the size and complexity of the problem domain and the minimum time step that can be used to solve such problems. One approach to address the computational burden associated with large-scale problems is to use DD, which decomposes the original problem domain into smaller subdomains that can be solved independently and then coupled together. The DD approach is frequently employed for coupled systems. Felippa et al. [33] provide an introduction to methods for solving coupled dynamical systems via partitioned analysis. Felippa and Park [34] also developed staggered solution procedures for second-order differential equations. Another example of the use of DD for solving coupled systems is of Farhat et al. [35], who studied loosely coupled nonlinear fluid-structure interaction on moving grids.

DD methods by themselves, however, do not address the computational burden associated with time integration of the subdomains for transient problems. In many cases, the accuracy and stability requirements within a specific subdomain, such as a subdomain containing a crack, necessitates a much smaller mesh spacing and time step than would otherwise be required in the other subdomains, leading to high computational costs. MTS methods allow relaxation of the temporal restrictions in subdomains that do not require 
such small time steps, while still retaining the accuracy of the original undecomposed model overall. Choosing time steps that are tailored to individual subdomains can significantly lower the computational cost of long-time simulations of large-scale problems.

Belytschko and coworkers [36, 37, 38, 39] proposed one of the earliest applications for differing time steps with multiple subdomains. Hughes and Liu $[40,41]$ used element partitioning to develop implicit-explicit finite elements. An explicit MTS method by Smolinski [42] addressed second order differential equations of structural dynamics, with a stability proof given in another paper [43]. Daniel [44, 45, 46] also developed different MTS extensions for the Newmark method. An implicit-explicit partitioning of a structural finite element mesh was investigated by Sotelino [47].

More recently, MTS methods have used Lagrange multipliers to couple different subdomains across a non-overlapping interface ensuring continuity of the solutions across the subdomain boundaries. Farhat and coworkers $[48,49]$ extended their 'finite element tearing and interconnecting' (FETI) method to dynamics. Gravouil and Combescure $[50,51]$ further extended the FETI method and showed that imposing continuity of discretized velocities at the interface led to a stable algorithm. Prakash and coworkers [17, 19, 18] improved upon the method of Gravouil and Combescure to eliminate numerical dissipation, preserve the accuracy of the subdomain solutions, and carry out the computations in an efficient manner.

Applications in modeling of fracture have benefited from the developments in DD and MTS methods. An area of interest, such as one containing a crack, is generally enclosed in a separate subdomain which can be modeled with a finer spatial and temporal discretization. The subdomain surrounding the cracked area is often modeled using an extended finite element method (see Guidault and coworkers [52, 53], and Wyart and coworkers [54, 55]). Researchers have also used DD to enable the use of an atomistic domain at the crack tip while using a continuum domain elsewhere (see Xiao and 
Belytschko [56] and Rafii-Tabar et al. [57]). DD and MTS methods have been used for modeling brittle materials using enhanced FETI techniques by Lloberas-Valls et al. [58], coupling finite elements and meshless methods by Belytschko et al. [59], and DD for nonlocal problems by Aksoylu and Parks [60], amongst others.

\section{A Multi-Time-Step Method for Peridynamics}

In the discretized PD approach, each discretized volumetric region is represented by a cell in a PD grid for which the governing equation of motion is given by (6). In many $\mathrm{PD}$ simulations, the accuracy and stability requirements of a relatively small number of cells dictate the time step for the entire domain. Often, there is neither the need nor the resources to solve the entire domain at the level of fidelity that is required in that small region. For example, Figure 2 shows an initially cracked plate loaded suddenly with tractions to induce Mode-I fracture at the crack tip. In such situations, DD can be used to divide the entire problem domain into different subdomains by grouping cells with similar accuracy and stability requirements together into one subdomain. For instance, in Figure 2, the region around the crack tip $\left(\Omega^{B}\right)$ may be enriched with a refined spatial discretization, while a coarser discretization may be used for the rest of the problem domain $\left(\Omega^{A}\right)$. In addition to DD, a MTS method can be employed to integrate subdomain $B$ with a small time step $\Delta t^{B}$, whereas subdomain $A$ may be integrated at a relatively larger time step $\Delta t^{A}$ for better computational efficiency, as depicted in Figure 3.

Due to the nonlocal nature of PD, several aspects of the existing DD and MTS methods must necessarily be modified and new concepts developed to enable a MTS-PD approach. For instance, the fundamental concept of an interface between subdomains needs to be generalized to an overlapping volumetric interface region $\Omega^{I}$ as shown in Figure 2. Aksoylu and Parks [60] found that unlike the non-overlapping sharp interfaces used in conventional 


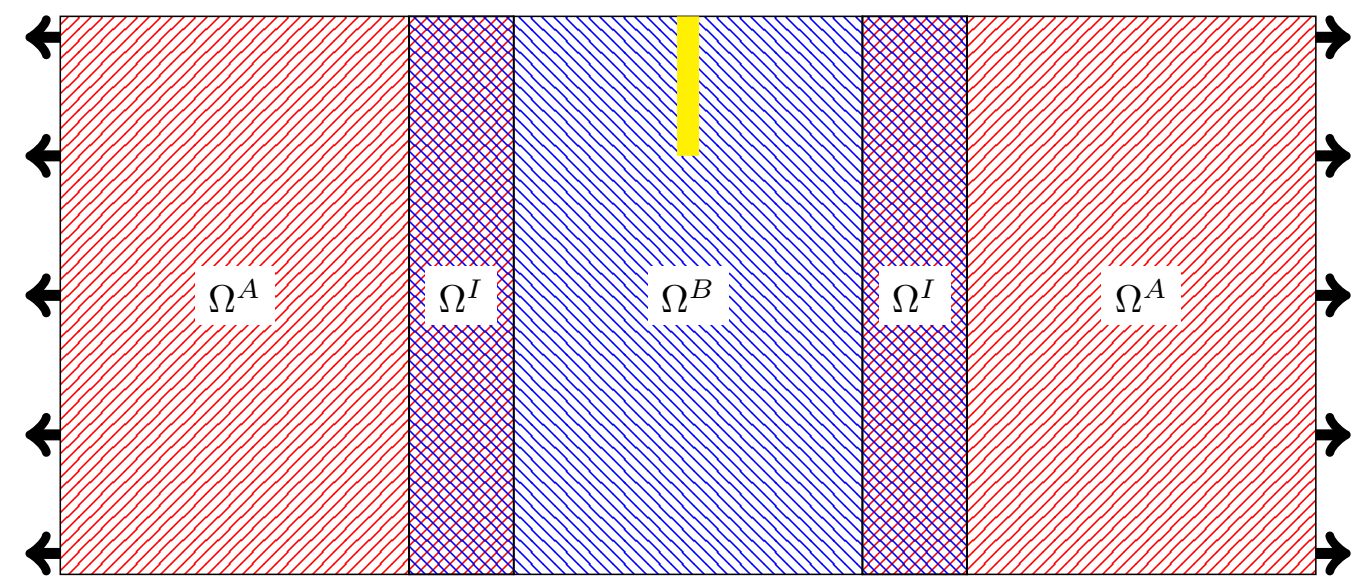

Figure 2: A two subdomain plate with an initial crack, loaded in tension, were the overlap subdomain $\Omega^{I}$ is defined as $\Omega^{I}=\Omega^{A} \cap \Omega^{B}$

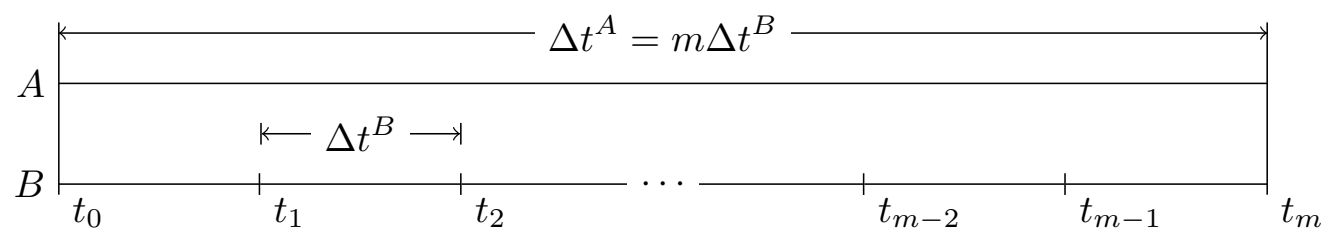

Figure 3: Time steps for two subdomains with a time-step ratio $m$ 
DD methods for solid mechanics, an overlapping volumetric interface region is needed for the non-local PD formulation. Further, for bond-based peridynamic models this overlapping volumetric interface region must be of a width that is at least equal to the horizon $\delta$, similar to the volumetric region used for implementation of boundary conditions. While this study focuses on the coupling of two nonlocal subdomains, MTS could also be applied to local-nonlocal coupling as described in Seleson et al. [61]. In the present work, Lagrange multipliers were used to enforce the continuity of velocities over this overlapping interface region and to couple the subdomains. This basic change in how the interface is defined leads to cascading changes in the entire MTS formulation, as discussed next.

For simplicity of derivation, the proposed MTS-PD method is presented for the case of two PD subdomains, $\Omega^{A}$ and $\Omega^{B}$, sharing a set of volumetric interface cells $\Omega^{I}=\Omega^{A} \cap \Omega^{B}$. Subdomains $A$ and $B$ are integrated with time steps $\Delta t^{A}$ and $\Delta t^{B}$ respectively, where $\Delta t^{A}=m \Delta t^{B}$ for some integer time-step ratio $m$, as shown in Figure 3. It is important to note that the MTS-PD approach naturally extends to a general number of subdomains and associated time steps.

In order to advance the solution in time, the state of the system must be advanced from some known state at $t_{n}$ to $t_{n+m}=t_{n}+\Delta t^{A}=t_{n}+m \Delta t^{B}$. For simplicity, these time instants are denoted as $t_{0}$ and $t_{m}$. The intermediate time instants between $t_{0}$ and $t_{m}$ will be denoted as $t_{j}=t_{0}+j \Delta t^{B}, j=$ $1,2, \ldots, m$. To solve, one must first advance subdomain $A$ one time step $\Delta t^{A}$ and subdomain $B m$ time steps $\Delta t^{B}$ using conventional PD computations. Lagrange multipliers for coupling, which are applied as body forces, can now be calculated. This is accomplished by enforcing the constraints that momentum is conserved across the volumetric interface region at the intermediate time steps $t_{j}$, and that the velocities of subdomains $A$ and $B$ are equal over the same interface region at the final time step $t_{m}$. Finally, the uncoupled solutions for both subdomains are updated using corrections 
derived from the Lagrange multipliers.

In practice, the kinematic values must be calculated for all cells $i$ in subdomain $B\left(\mathcal{C}^{i} \subset \Omega^{B}\right)$ at each time instant $t_{j}, j=1,2, \ldots, m$. For cells $i$ in subdomain $A$ that are not in the interface region $\left(\mathcal{C}^{i} \subset \Omega^{A} \backslash \Omega^{I}\right)$, the kinematic values need only be calculated at the common instant in time $t_{m}$. Additionally, for each cell $i$ in the interface region $\left(\mathcal{C}^{i} \subset \Omega^{I}\right)$ a Lagrange multiplier $\lambda$ must be included in the equations of motion for both subdomains $A$ and $B$ to couple them together. In this sense, the Lagrange multipliers can be interpreted as interface forces acting internally between the subdomains to maintain continuity of the solutions across the interface.

Thus, the peridynamic equations of motion for each cell will take the form

$$
\begin{aligned}
& \rho^{A} \boldsymbol{a}_{m}^{A, i}=\sum_{\boldsymbol{x}^{p} \in \mathcal{H}_{\boldsymbol{H}^{A}}^{A}} \boldsymbol{C}^{A}\left(\boldsymbol{x}^{A, p}-\boldsymbol{x}^{A, i}\right)\left(\boldsymbol{d}_{m}^{A, p}-\boldsymbol{d}_{m}^{A, i}\right) V^{p}+\boldsymbol{b}_{m}^{A, i}+c^{A, i} \boldsymbol{\lambda}_{m}^{i} \\
& \rho^{B} \boldsymbol{a}_{j}^{B, i}=\sum_{\boldsymbol{x}^{p} \in \mathcal{H}_{\boldsymbol{H}^{i}}^{B}} \boldsymbol{C}^{B}\left(\boldsymbol{x}^{B, p}-\boldsymbol{x}^{B, i}\right)\left(\boldsymbol{d}_{j}^{B, p}-\boldsymbol{d}_{j}^{B, i}\right) V^{p}+\boldsymbol{b}_{j}^{B, i}+c^{B, i} \boldsymbol{\lambda}_{j}^{i}, \forall j=1,2, \ldots, m,
\end{aligned}
$$

where $\mathcal{H}_{x^{i}}^{k}=\mathcal{H}_{x^{i}} \cap \Omega^{k}$ is the set of all material points in subdomain $k$ which are also in the horizon of $\boldsymbol{x}^{i} \in \Omega^{k}$. For cells $i$ in subdomain $k$ that are not in the interface region $\left(\mathcal{C}^{i} \subset \Omega^{k} \backslash \Omega^{I}\right)$, the density $\rho^{k}$ in subdomain $k$ is simply the uniform material density $\rho$. For cells in the overlapping interface region $\left(\mathcal{C}^{i} \subset \Omega^{I}\right), \rho^{k}$ represents a fraction of the total density such that $\rho^{A}+\rho^{B}=\rho$ (see (6)). Although it is not required, a convenient distribution of densities in the interface region is $\rho^{A}=\rho^{B}=1 / 2 \rho$. The body force is distributed similarly, such that $\boldsymbol{b}_{j}^{A, i}+\boldsymbol{b}_{j}^{B, i}=\boldsymbol{b}_{j}^{i}$ for interface cells. Likewise, the micromodulus is distributed such that $\boldsymbol{C}^{A}+\boldsymbol{C}^{B}=\boldsymbol{C}$ if both cells $i$ and $p$ are in the interface, and $\boldsymbol{C}^{A}=\boldsymbol{C}^{B}=\boldsymbol{C}$ otherwise. For cells in the interface region, the coefficients $c^{k, i}$ have values of $c^{A, i}=1, c^{B, i}=-1$. Elsewhere, they are zero.

The peridynamic equations of motion for all cells in a certain subdomain 
$k$ can be generalized as

$$
\boldsymbol{M}^{k} \boldsymbol{a}_{j}^{k}+\boldsymbol{K}^{k} \boldsymbol{d}_{j}^{k}+\Gamma^{k^{T}} \boldsymbol{\lambda}_{j}=\boldsymbol{b}_{j}^{k}
$$

where the vectors shown are of the form $\boldsymbol{x}_{j}^{k}=\left\{\boldsymbol{x}_{j}^{k, 1}, \boldsymbol{x}_{j}^{k, 2}, \ldots, \boldsymbol{x}_{j}^{k, N}\right\}^{T}$, and $N=n^{k}$ is the number of cells in subdomain $k$. Additionally, the interface reaction force density vector $\boldsymbol{\lambda}_{j}$ is defined over the interface cells $\mathcal{C}^{i} \subset \Omega^{I}$ as $\boldsymbol{\lambda}_{j}=\left\{\boldsymbol{\lambda}_{j}^{i_{1}}, \boldsymbol{\lambda}_{j}^{i_{2}}, \ldots, \boldsymbol{\lambda}_{j}^{i_{N}}\right\}^{T}$, where $N=n^{I}$ is the number of cells in the interface. The matrices $\boldsymbol{M}^{k}, \boldsymbol{K}^{k}$, and $\boldsymbol{\Gamma}^{k}$ can be defined in component form as follows:

$$
\begin{gathered}
{\left[M^{k}\right]_{i j}=\rho^{k} \delta_{i j}= \begin{cases}\rho^{k} & i=j \\
0 & \text { otherwise }\end{cases} } \\
{\left[K^{k}\right]_{i j}= \begin{cases}\sum_{\boldsymbol{x}^{p} \in \mathcal{H}^{k}} \boldsymbol{C}^{k}\left(\boldsymbol{x}^{k, p}-\boldsymbol{x}^{k, i}\right) V^{p} & i=j \\
-\boldsymbol{C}^{k}\left(\boldsymbol{x}^{k, j}-\boldsymbol{x}^{k, i}\right) V^{j} & \mathcal{C}^{j} \subset \mathcal{H}_{\boldsymbol{x}^{i}}^{k}, i \neq j \\
0 & \text { otherwise }\end{cases} } \\
{\left[\Gamma^{k}\right]_{i j}=-c^{k, j} \delta_{i j},}
\end{gathered}
$$

where $i$ and $j$ are two cells in subdomain $k$. Note that while we focus on bond-based models in this work, (13) generalizes to state-based PD material models as well. For such models, interactions would occur over $2 \delta$ as opposed to $\delta$ for bond-based models and (13) would remain largely unchanged.

As with undecomposed PD, the Newmark- $\beta$ algorithm is used to advance the solution forward in time, but with the different subdomains advancing at possibly different time steps using different Newmark- $\beta$ schemes. The choice of time steps and time-integration schemes for individual subdomains can be made based on the same criteria that are used for undecomposed PD domains mentioned in section 2.4. For a more detailed discussion of how this choice can be made for MTS-PD methods, one may refer to section 4.3 of [18]. 
The Newmark- $\beta$ equations can be generalized for all cells within a subdomain as

$$
\begin{aligned}
& \boldsymbol{v}_{m}^{A}=\boldsymbol{v}_{0}^{A}+\Delta t^{A}\left[\left(1-\gamma^{A}\right) \boldsymbol{a}_{0}^{A}+\gamma^{A} \boldsymbol{a}_{m}^{A}\right] \\
& \boldsymbol{d}_{m}^{A}=\boldsymbol{d}_{0}^{A}+\Delta t^{A} \boldsymbol{v}_{0}^{A}+\Delta t^{A^{2}}\left[\left(1 / 2-\beta^{A}\right) \boldsymbol{a}_{0}^{A}+\beta^{A} \boldsymbol{a}_{m}^{A}\right]
\end{aligned}
$$

and

$$
\begin{aligned}
& \boldsymbol{v}_{j}^{B}=\boldsymbol{v}_{j-1}^{B}+\Delta t^{B}\left[\left(1-\gamma^{B}\right) \boldsymbol{a}_{j-1}^{B}+\gamma^{B} \boldsymbol{a}_{j}^{B}\right] \\
& \boldsymbol{d}_{j}^{B}=\boldsymbol{d}_{j-1}^{B}+\Delta t^{B} \boldsymbol{v}_{j-1}^{B}+\Delta t^{B^{2}}\left[\left(1 / 2-\beta^{B}\right) \boldsymbol{a}_{j-1}^{B}+\beta^{B} \boldsymbol{a}_{j}^{B}\right] .
\end{aligned}
$$

The equations of motion and the Newmark- $\beta$ equations account for the kinematic quantities of subdomain $B$ at all time steps $j=1,2, \ldots, m$, and of subdomain $A$ at the final step $m$. However, subdomain $A$ cells in the interface $\left(\mathcal{C}^{i} \subset \Omega^{I}\right)$ must also have kinematic values defined at the intermediate time steps $j=1,2, \ldots, m-1$. Thus, the intermediate kinematic values of the cells in subdomain $A$ are linearly interpolated from the known values at the common time steps $j=0$ and $m$. Specifically,

$$
\boldsymbol{z}_{j}^{A, i}=(1-j / m) \boldsymbol{z}_{0}^{A, i}+(j / m) \boldsymbol{z}_{m}^{A, i}, j=1,2, \ldots, m-1
$$

where $\boldsymbol{z}_{j}^{A, i} \equiv\left\{\boldsymbol{d}_{j}^{A, i}, \boldsymbol{v}_{j}^{A, i}, \boldsymbol{a}_{j}^{A, i}\right\}^{T}$.

To balance $\boldsymbol{\lambda}_{m}$, the interface reaction force density at the common time step, the continuity of a kinematic quantity must be enforced on the interface boundary. Previous research on classical MTS and Newmark- $\beta$ schemes has shown that continuity of velocities at the interface is the only choice for continuity of a kinematic quantity that leads to unconditionally stable results (see [50]). Numerical results for the MTS-PD approach also support this result, and continuity of velocity is the constraint that was used here as well. This leads to an additional set of velocity continuity equations,

$$
c^{A, i} \boldsymbol{v}_{m}^{A, i}+c^{B, i} \boldsymbol{v}_{m}^{B, i}=0
$$


for each interface cell $\left(\mathcal{C}^{i} \subset \Omega^{I}\right)$, or

$$
\Gamma^{A} \boldsymbol{v}_{m}^{A}+\Gamma^{B} \boldsymbol{v}_{m}^{B}=0
$$

for all cells.

Additional equations are also required to balance the interface reaction force densities $\boldsymbol{\lambda}_{j}$ at the intermediate time steps $j=1,2, \ldots, m-1$. For this, conservation of momentum equations were used:

$\rho^{A} \boldsymbol{a}_{j}^{A, i}=\sum_{\boldsymbol{x}^{p} \in \mathcal{H}_{\boldsymbol{x}^{i}}^{A}} \boldsymbol{C}^{A}\left(\boldsymbol{x}^{A, p}-\boldsymbol{x}^{A, i}\right)\left(\boldsymbol{d}_{j}^{A, p}-\boldsymbol{d}_{j}^{A, i}\right) V^{p}+\boldsymbol{b}_{j}^{A, i}+c^{A, i} \boldsymbol{\lambda}_{j}^{i}, \forall j=1,2, \ldots, m-1$

for each interface cell $\left(\mathcal{C}^{i} \subset \Omega^{I}\right)$, or

$$
\boldsymbol{\Gamma}^{A}\left[\boldsymbol{M}^{A} \boldsymbol{a}_{j}^{A}+\boldsymbol{K}^{A} \boldsymbol{d}_{j}^{A}+\boldsymbol{\Gamma}^{A^{T}} \boldsymbol{\lambda}_{j}-\boldsymbol{b}_{j}^{A}\right]=\mathbf{0}
$$

for all cells.

The above equations can be expressed more compactly using the following definitions:

$$
\mathbb{M}^{k}=\left[\begin{array}{ccc}
\boldsymbol{M}^{k} & \mathbf{0} & \boldsymbol{K}^{k} \\
-\gamma^{k} \Delta t^{k} \boldsymbol{I} & \boldsymbol{I} & \mathbf{0} \\
-\beta^{k} \Delta t^{k} \boldsymbol{I} & \mathbf{0} & \boldsymbol{I}
\end{array}\right], \mathbb{N}^{k}=\left[\begin{array}{ccc}
\mathbf{0} & \mathbf{0} & \mathbf{0} \\
-\left(1-\gamma^{k}\right) \Delta t^{k} \boldsymbol{I} & -\boldsymbol{I} & \mathbf{0} \\
-\left(1 / 2-\beta^{k}\right) \Delta t^{k^{2}} \boldsymbol{I} & -\Delta t^{k} \boldsymbol{I} & -\boldsymbol{I}
\end{array}\right]
$$

where $\mathbb{M}^{k}$ and $\mathbb{N}^{k}$ are block matrices for each subdomain $k$.

With these definitions, (13), (17) and (18) can now be combined into the following general equations:

$$
\begin{aligned}
\mathbb{M}^{A} \mathbb{U}_{m}^{A}+\mathbb{N}^{A} \mathbb{U}_{0}^{A}+\mathbb{C}^{A} \boldsymbol{\lambda}_{m} & =\mathbb{P}_{m}^{A} \\
\mathbb{M}^{B} \mathbb{U}_{j}^{B}+\mathbb{N}^{B} \mathbb{U}_{j-1}^{B}+\mathbb{C}^{B} \boldsymbol{\lambda}_{j} & =\mathbb{P}_{j}^{B}, \forall j=1,2, \ldots, m,
\end{aligned}
$$


with block vectors $\mathbb{C}^{k}, \mathbb{U}_{j}^{k}$, and $\mathbb{P}_{j}^{k}$ defined as

$$
\mathbb{C}^{k}=\left[\begin{array}{c}
\Gamma^{k^{T}} \\
\mathbf{0} \\
\mathbf{0}
\end{array}\right], \mathbb{U}_{j}^{k}=\left[\begin{array}{c}
\boldsymbol{a}_{j}^{k} \\
\boldsymbol{v}_{j}^{k} \\
\boldsymbol{d}_{j}^{k}
\end{array}\right], \mathbb{P}_{j}^{k}=\left[\begin{array}{c}
\boldsymbol{b}_{j}^{k} \\
\mathbf{0} \\
\mathbf{0}
\end{array}\right]
$$

for each subdomain $k$.

As an alternative to direct solution, the equations given above can be solved more efficiently by decoupling each subdomain and solving them separately (see section 4.1 of [17]). The first step of this procedure is solving

$$
\mathbb{M}^{A} \mathbb{V}_{m}^{A}+\mathbb{N}^{A} \mathbb{U}_{0}^{A}=\mathbb{P}_{m}^{A}
$$

which is equivalent to advancing subdomain $A$ independently one time step $\Delta t^{A}$ under external loads. Subdomain $B$ is also advanced independently for $m$ time steps $\Delta t^{B}$ under external loads and free interface reactions $\boldsymbol{S}_{j}$,

$$
\mathbb{M}^{B} \mathbb{V}_{j}^{B}+\mathbb{N}^{B} \mathbb{V}_{j-1}^{B}=\mathbb{P}_{j}^{B}-\mathbb{C}^{B} \boldsymbol{S}_{j}, \forall j=1,2, \ldots, m
$$

where $\mathbb{V}_{0}^{B}=\mathbb{U}_{0}^{B}$ and $\boldsymbol{S}_{j}=(1-j / m) \boldsymbol{\lambda}_{0}+\boldsymbol{\Gamma}^{A}\left[\boldsymbol{b}_{j}^{A}-(1-j / m) \boldsymbol{b}_{0}^{A}-(j / m) \boldsymbol{b}_{m}^{A}\right]$.

Another necessary intermediate result is obtained by solving

$$
\mathbb{M}^{A} \mathbb{Y}_{m}^{A}=\mathbb{C}^{A}
$$

which is equivalent to applying a unit load to one shared cell at a time and advancing subdomain $A$ independently for one time step $\Delta t^{A}$. A unit load is also applied to each shared cell in subdomain $B$ and the solution is advanced independently for $m$ time steps $\Delta t^{B}$,

$$
\mathbb{M}^{B} \mathbb{Y}_{j}^{B}+\mathbb{N}^{B} \mathbb{Y}_{j-1}^{B}=(j / m) \mathbb{C}^{B}, \forall j=1,2, \ldots, m
$$

where $\mathbb{Y}_{0}^{B}=\mathbf{0}$. For efficiency, (29) and (30) should be calculated once at the 
beginning of a simulation and need not be recalculated until bond breakage occurs.

Finally, the vector of interface reaction force densities $\boldsymbol{\lambda}_{m}$ can be determined at the common time step $m$ using the following relation:

$$
\left[\mathbb{C}^{A} \dot{\boldsymbol{Y}}_{m}^{A}+\mathbb{C}^{B} \dot{\boldsymbol{Y}}_{m}^{B}\right] \boldsymbol{\lambda}_{m}=\mathbb{C}^{A} \dot{\boldsymbol{V}}_{m}^{A}+\mathbb{C}^{B} \dot{\boldsymbol{V}}_{m}^{B}
$$

where the $\dot{\boldsymbol{Y}}_{m}^{A}, \dot{\boldsymbol{Y}}_{m}^{B}, \dot{\boldsymbol{V}}_{m}^{A}$ and $\dot{\boldsymbol{V}}_{m}^{B}$ matrices are obtained from the velocity components of their corresponding block matrices $\mathbb{Y}_{m}^{A}, \mathbb{Y}_{m}^{B}, \mathbb{V}_{m}^{A}$ and $\mathbb{V}_{m}^{B}$ respectively.

The final coupled results can now be obtained from the following equations:

$$
\mathbb{U}_{m}^{A}=\mathbb{V}_{m}^{A}-\mathbb{Y}_{m}^{A} \boldsymbol{\lambda}_{m}, \mathbb{U}_{j}^{B}=\mathbb{V}_{j}^{B}-\mathbb{Y}_{j}^{B} \boldsymbol{\lambda}_{m}
$$

which is mathematically equivalent to solving the coupled monolithic system (25), but is substantially less expensive. The pseudocode for the MTS-PD method is shown in Algorithm 1.

\section{Evaluation of Multi-Time-Step Peridynamics}

\subsection{Computational Cost Analysis}

In this section we compare the computational cost of the MTS-PD method against the cost of the undecomposed PD method using Newmark- $\beta$ with a single timestep. First, it is noted that the cost of advancing the model by a single timestep for both explicit and implicit methods is dominated by the time it takes to evaluate bonds, not by matrix-vector operations or factorizations needed for the time-stepping scheme. Thus the computational cost of a model is proportional to the total number of bonds in the discrete system. There are a given number of initially unbroken bonds connecting any interior cell to its surrounding cells. This number is based on the horizon of the system. For example, if the horizon is three times the mesh spacing, there will be 122 bonds on each interior cell. For four times the mesh spacing, that 


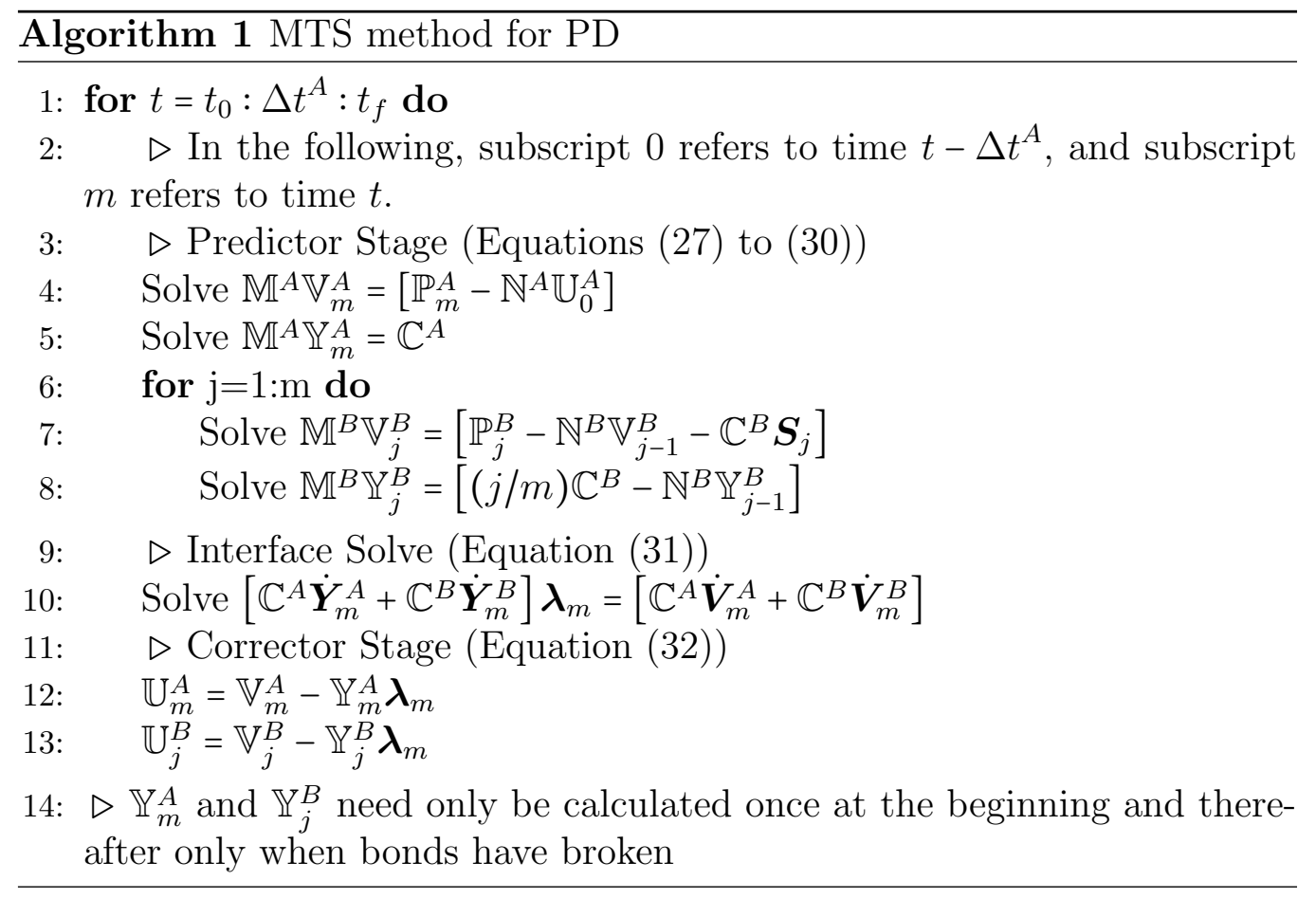

number grows to 256, while for two, there are only 32 bonds. This comes from the fact that the horizon can be thought of as the radius of a sphere surrounding a cell. The number of bonds can in turn be used to estimate the computational benefits of utilizing a MTS scheme with PD.

The number of bonds at each interior cell is denoted by $n_{b}$. Estimating the computational costs for all cells based on this value yields a relatively simple method for comparing the relative costs of undecomposed PD and MTS-PD. This is accomplished by approximating the total number of bonds to be evaluated in each large time step. Taking $n$ as the number of cells in the system and $m=\Delta t^{A} / \Delta t^{B}$ as the time-step ratio, the undecomposed PD system will have approximately $n_{b} n m$ bonds to evaluate in one large time step $\Delta t^{A}$. For the split system, the total number of cells can be distributed as $n=n^{A}+n^{B}+n^{I}$, where $n^{A}, n^{B}$, and $n^{I}$ are the number of cells exclusively in each subdomain $\left(\Omega^{A} \backslash \Omega^{I}\right.$ and $\left.\Omega^{B} \backslash \Omega^{I}\right)$ and in the overlap between the 
subdomains $\left(\Omega^{I}\right)$ respectively.

Using these definitions, the approximate number of bonds in the split system to be evaluated in one large time step is $n_{b}\left(n^{A}+\left(n^{B}+2 n^{I}\right) m\right)$. This indicates that when $n^{A}(m-1)>m n^{I}$, splitting a PD domain into two subdomains as outlined previously will be cost effective. It is also useful to note that the relative cost savings between MTS applied to classical mechanics and nonlocal PD should be approximately equal, since the estimate is independent of $n_{b}$. This is because an equivalent cost analysis for the classical method would yield the same results as PD with a horizon $\delta=\Delta x$. Note: In general, the horizon $\delta$ is assumed to be small with respect to domain size.

For a large system, the benefits of using MTS over an undecomposed system become immediately clear. For a system 100 cells wide and a timestep ratio of 2 , with subdomain $B$ being $10 \%$ of the total width, the simple cost analysis shown earlier indicates that the split system will be about $50 \%$ faster than the undecomposed system. For 1000 cells and the same width of subdomain $B$, that number grows to $90 \%$, and much higher with larger time-step ratios.

\subsection{Stability Analysis}

In this section, we show that the MTS-PD method inherits the stability properties of the MTS method for classical solid mechanics. Following along the lines of the stability proof given in [17], the stability analysis of the MTS$\mathrm{PD}$ method can be conducted to show that the change in energy for every large time step $\Delta t^{A}$ is

$$
\begin{aligned}
\mathcal{E}= & -\left(\gamma^{A}-1 / 2\right)\left(\boldsymbol{a}_{m}^{A}-\boldsymbol{a}_{0}^{A}\right)^{T} \boldsymbol{A}^{A}\left(\boldsymbol{a}_{m}^{A}-\boldsymbol{a}_{0}^{A}\right) \\
& -\sum_{j=1}^{m}\left(\gamma^{B}-1 / 2\right)\left(\boldsymbol{a}_{j}^{B}-\boldsymbol{a}_{j-1}^{B}\right)^{T} \boldsymbol{A}^{B}\left(\boldsymbol{a}_{j}^{B}-\boldsymbol{a}_{j-1}^{B}\right)+E^{\lambda},
\end{aligned}
$$

where

$$
\boldsymbol{A}^{k}=\boldsymbol{M}^{k}+\Delta t_{k}^{2}\left(\beta^{k}-\frac{\gamma^{k}}{2}\right) \boldsymbol{K}^{k}
$$


and $k$ denotes the subdomain number.

For a method to be numerically stable, the change in energy $\mathcal{E}$ must be less than or equal to zero. For the bond-based PD model considered here, the mass and stiffness matrices $\boldsymbol{M}^{k}$ and $\boldsymbol{K}^{k}$, and consequently $\boldsymbol{A}^{k}$, remain positive definite, as long as the individual subdomains are stable. Thus the stability of the MTS-PD method is governed by $E^{\lambda}$, which is expressed as

$$
\begin{aligned}
E^{\lambda}= & 1 / \Delta t^{A}\left(\boldsymbol{v}_{m}^{A}-\boldsymbol{v}_{0}^{A}\right)^{T} \boldsymbol{\Gamma}^{A}\left(\boldsymbol{\lambda}_{m}^{A}-\boldsymbol{\lambda}_{0}^{A}\right) \\
& +1 / \Delta t^{B} \sum_{j=1}^{m}\left(\boldsymbol{v}_{j}^{B}-\boldsymbol{v}_{j-1}^{B}\right)^{T} \boldsymbol{\Gamma}^{B}\left(\boldsymbol{\lambda}_{j}^{B}-\boldsymbol{\lambda}_{j-1}^{B}\right) .
\end{aligned}
$$

This term can be shown to be identically zero due to the continuity of velocities at the interface, as enforced by the constraint in (21). Consequently, the coupled method is stable as long as both $\gamma^{A}$ and $\gamma^{B}$ are greater than or equal to $1 / 2$. For more details on the stability proof, see [17].

\subsection{Error Analysis}

In this section, we show that the MTS-PD method inherits the order of accuracy of the Newmark- $\beta$ method that is applied to each subdomain. As previously mentioned, the simplest method of solving the split system is to combine the set of equations into a linear system of equations. This yields a state space representation of the following form:

$$
\boldsymbol{A}_{1} \mathbb{\mathrm { Y }}=\boldsymbol{A}_{2} \boldsymbol{y}_{0}+\boldsymbol{c}_{0}
$$

where $\boldsymbol{A}_{1}$ and $\boldsymbol{A}_{2}$ are coefficient matrices and $\boldsymbol{c}_{0}$ is a vector containing the body force for rows representing equations of motion and zero elsewhere. The vectors $\boldsymbol{y}_{0}$ and $\mathbb{y}$ contain the kinematic values at the current and next time steps respectively, and are given by

$$
\mathbb{y}=\left\{\boldsymbol{z}_{m}^{A}, \boldsymbol{z}_{1}^{B}, \ldots, \boldsymbol{z}_{m}^{B}, \boldsymbol{\lambda}_{1}, \ldots, \boldsymbol{\lambda}_{m}\right\}^{T}, \boldsymbol{y}_{0}=\left\{\boldsymbol{z}_{0}^{A}, \boldsymbol{z}_{0}^{B}, \boldsymbol{\lambda}_{0}\right\}^{T},
$$


where the vector $\boldsymbol{z}_{j}^{k}$ is given by

$$
\boldsymbol{z}_{j}^{k} \equiv\left\{\boldsymbol{z}_{j}^{k, 1}, \boldsymbol{z}_{j}^{k, 2}, \ldots, \boldsymbol{z}_{j}^{k, n}\right\}^{T}
$$

at each time step $j$, where $n$ represents the total number of cells in subdomain $k$. The vector $\boldsymbol{z}_{j}^{k, i}$ is further defined for each subdomain $k$ as $\boldsymbol{z}_{j}^{k, i} \equiv$ $\left\{\boldsymbol{d}_{j}^{k, i}, \boldsymbol{v}_{j}^{k, i}, \boldsymbol{a}_{j}^{k, i}\right\}^{T}$.

Solving for the unknown kinematic variables directly will result in a set of equations of the form

$$
\mathbb{y}=\boldsymbol{A} \boldsymbol{y}_{0}+\boldsymbol{l}_{0}
$$

where $\boldsymbol{A}=\boldsymbol{A}_{1}^{-1} \boldsymbol{A}_{2}$ and $\boldsymbol{l}_{0}=\boldsymbol{A}_{1}^{-1} \boldsymbol{c}_{0}$.

A quantity of interest is the local truncation error, $\boldsymbol{\tau}\left(t_{n}\right)$, which is the error accrued in one step of the solution procedure. This is equivalent to the error in the approximate solution, $\boldsymbol{y}_{n+1}-\boldsymbol{y}\left(t_{n+1}\right)$, when taking one step in time and using exact initial data.

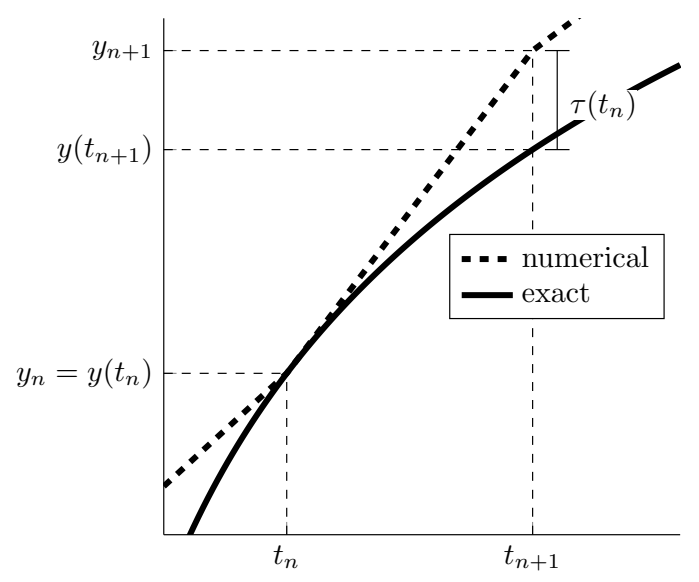

Figure 4: Local truncation error represented as the error in the approximate solution

The truncation error can be related to the state space form of the system of equations using exact kinematic values as

$$
\mathbb{y}\left(t_{\star}\right)=\boldsymbol{A} \boldsymbol{y}\left(t_{0}\right)+\boldsymbol{l}\left(t_{0}\right)+\boldsymbol{\tau}\left(t_{0}\right)
$$


where $\boldsymbol{\tau}\left(t_{0}\right)$ represents the local truncation error. Note: $\mathbb{y}\left(t_{*}\right)$ and $\boldsymbol{y}\left(t_{0}\right)$, which are both vectors of exact values, are analogs of $\mathbb{y}$ and $\boldsymbol{y}_{0}$, which are vectors of approximate values. Specifically,

$$
\begin{aligned}
\mathbb{y}\left(t_{*}\right) & =\left\{\boldsymbol{z}^{A}\left(t_{m}\right), \boldsymbol{z}^{B}\left(t_{1}\right), \ldots, \boldsymbol{z}^{B}\left(t_{m}\right), \boldsymbol{\lambda}\left(t_{1}\right), \ldots, \boldsymbol{\lambda}\left(t_{m}\right)\right\}^{T}, \\
\boldsymbol{y}\left(t_{0}\right) & =\left\{\boldsymbol{z}^{A}\left(t_{0}\right), \boldsymbol{z}^{B}\left(t_{0}\right), \boldsymbol{\lambda}\left(t_{0}\right)\right\}^{T} .
\end{aligned}
$$

It is useful to reiterate that the truncation error is a local measure of error. To obtain global errors, the iterative relation

$$
\boldsymbol{\epsilon}\left(t_{n+m}\right)=\boldsymbol{\tau}\left(t_{n+m}\right)+\boldsymbol{A} \boldsymbol{\epsilon}\left(t_{n}\right)
$$

holds, where $\boldsymbol{\epsilon}\left(t_{n}\right)$ and $\boldsymbol{\epsilon}\left(t_{n+m}\right)$ are global errors at the current and next common time step respectively, and $\boldsymbol{A}$ is taken from (39). This relation is obtained by comparing the local truncation error $\boldsymbol{\tau}\left(t_{m}\right)$ to the global truncation error $\boldsymbol{\epsilon}\left(t_{m}\right)=\mathbb{y}-\mathbb{y}\left(t_{*}\right)$. At time $t_{m}$ this leads to $\boldsymbol{\tau}\left(t_{m}\right)=\boldsymbol{\epsilon}\left(t_{m}\right)$, at time $t_{2 m}$ to $\boldsymbol{\epsilon}\left(t_{2 m}\right)=\boldsymbol{\tau}\left(t_{2 m}\right)+\boldsymbol{A} \boldsymbol{\epsilon}\left(t_{m}\right)$, and continuing on (42) is obtained.

The truncation error for these equations was explored analytically and numerically for a small decomposed PD system. The case chosen was a simple PD domain consisting of two cells, as shown in Figure 5. For the split system, cell 2 was taken to be in subdomain $B$, and both cells were solved in the manner outlined previously.

As a verification, both cells were integrated at the same time step $(m=1)$, with the same integration parameters $\gamma$ and $\beta$. Equal and opposite end forces, applied at each of the two nodes, were prescribed to induce sinusoidal response.

The complete set of equations to describe this system for one time step 


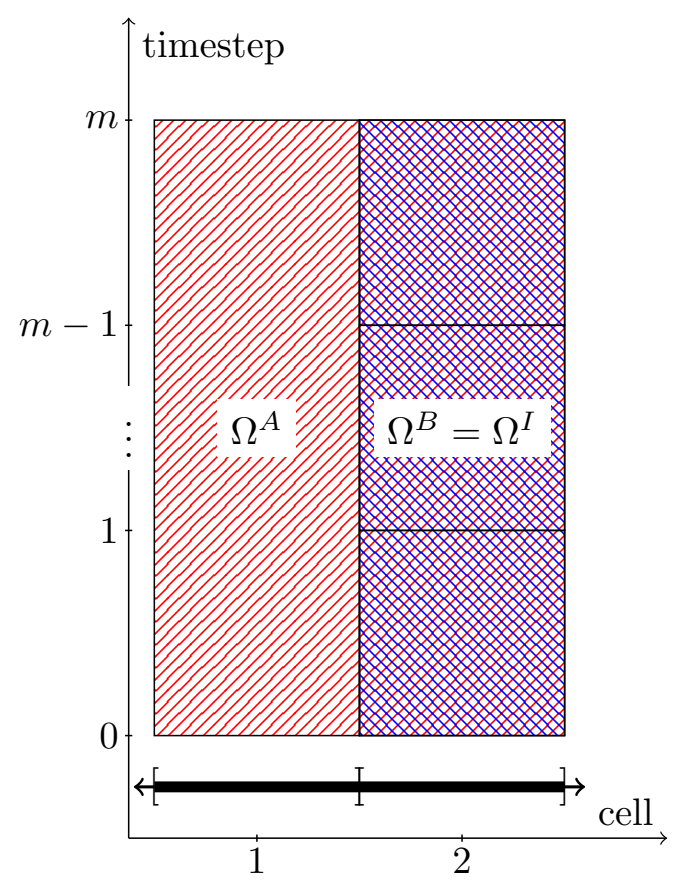

Figure 5: Space/time depiction of a one-dimensional, two-cell PD bar loaded in tension

$\Delta t$ and with horizon $\delta=\Delta x$ is given by

$$
\begin{gathered}
\rho a_{1}^{A, 1}=C\left(d_{1}^{A, 2}-d_{1}^{A, 1}\right) V^{2}+b_{1}^{A, 1} \\
\rho^{A} a_{1}^{A, 2}=C\left(d_{1}^{A, 1}-d_{1}^{A, 2}\right) V^{1}+\lambda_{1}^{2}+b_{1}^{A, 2} \\
\rho^{B} a_{1}^{B, 2}=-\lambda_{1}^{2}+b_{1}^{B, 2} \\
d_{1}^{k, i}=d_{0}^{k, i}+\Delta t v_{0}^{k, i}+\Delta t^{2}\left[(1 / 2-\beta) a_{0}^{k, i}+\beta a_{1}^{k, i}\right] \\
v_{1}^{k, i}=v_{0}^{k, i}+\Delta t\left[(1-\gamma) a_{0}^{k, i}+\gamma a_{1}^{k, i}\right] \\
v_{1}^{A, 2}=v_{1}^{B, 2}
\end{gathered}
$$

for subdomain $k=A$ at each cell $i=1,2$, and subdomain $k=B$ at cell $i=2$. Above, (43a) corresponds to the equations of motion, (43b) and (43c) to the Newmark displacement and velocity equations, and (43d) to the continuity of velocity equation. 
Before determining the truncation error, it is common to first substitute and eliminate acceleration. For the simple two cell system described above, this procedure results in the simplified set of state-space equations

$$
\boldsymbol{A}_{1} \boldsymbol{y}_{1}=\boldsymbol{A}_{2} \boldsymbol{y}_{0}+\boldsymbol{c}_{0}
$$

where $\boldsymbol{y}_{j}$, now given by $\boldsymbol{y}_{j}=\left\{d_{j}^{A, 1}, d_{j}^{A, 2}, d_{j}^{B, 2}, v_{j}^{A, 1}, v_{j}^{A, 2}, v_{j}^{B, 2}, \lambda_{j}^{2}\right\}^{T}(j=0,1)$, again represents a vector of approximate solutions.

Writing the equation in terms of the exact kinematic values and taking the truncation errors into account yields

$$
\boldsymbol{\tau}\left(t_{0}\right)=\boldsymbol{y}\left(t_{1}\right)-\boldsymbol{A} \boldsymbol{y}\left(t_{0}\right)-\boldsymbol{l}\left(t_{0}\right),
$$

which has a form similar to (40). Furthermore, $\boldsymbol{y}\left(t_{j}\right)$, an analog of $\boldsymbol{y}_{j}$, is given by

$$
\boldsymbol{y}\left(t_{j}\right)=\left\{u^{1}\left(t_{j}\right), u^{2}\left(t_{j}\right), u^{2}\left(t_{j}\right), \dot{u}^{1}\left(t_{j}\right), \dot{u}^{2}\left(t_{j}\right), \dot{u}^{2}\left(t_{j}\right), \lambda^{2}\left(t_{j}\right)\right\}^{T},
$$

and represents a vector of exact kinematic values (the true solution of the governing equations (43)). Note: the presence of repeated variables in (46) is due to the repetition of cells from subdomains $\Omega^{A}$ and $\Omega^{B}$ in $\Omega^{I}=\Omega^{A} \cap \Omega^{B}$.

To solve for the truncation error, each of the exact kinematic values can be expanded about two common points in time, $t_{2 \beta}=2 \beta t_{0}+(1-2 \beta) t_{1}$ and $t_{\gamma}=\gamma t_{0}+(1-\gamma) t_{1}$, using a Taylor series expansion. Both of these expansion points were chosen to aid in the simplification of the truncation error results.

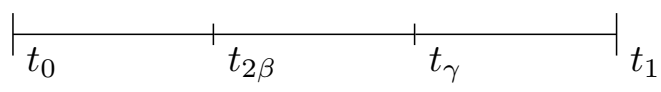

Figure 6: Taylor expansion points used to analyze the truncation error for a single time step

After substitution of (43) into (45), and assuming that $\rho^{A}=\rho^{B}=1 / 2 \rho$, 
$b_{1}^{A, 1}=b_{1}^{1}$, and $b_{2}^{A, 1}=b_{2}^{B, 1}=1 / 2 b_{1}^{2}$, the following matrix forms were obtained

$$
\begin{aligned}
& \boldsymbol{A}_{1}=\left[\begin{array}{ccccccc}
\frac{C V^{2} \beta \Delta t^{2}}{\rho}+1 & -\frac{C V^{2} \beta \Delta t^{2}}{\rho} & 0 & 0 & 0 & 0 & 0 \\
-\frac{2 C V^{1} \beta \Delta t^{2}}{\rho} & \frac{2 C V^{1} \beta \Delta t^{2}}{\rho}+1 & 0 & 0 & 0 & 0 & -\frac{2 \beta \Delta t^{2}}{\rho} \\
0 & 0 & 1 & 0 & 0 & 0 & \frac{2 \beta \Delta t^{2}}{\rho} \\
\frac{C V^{2} \gamma \Delta t}{\rho} & -\frac{C V^{2} \gamma \Delta t}{\rho} & 0 & 1 & 0 & 0 & 0 \\
-\frac{2 C V^{1} \gamma \Delta t}{\rho} & \frac{2 C V^{1} \gamma \Delta t}{\rho} & 0 & 0 & 1 & 0 & -\frac{2 \gamma \Delta t}{\rho} \\
0 & 0 & 0 & 0 & 0 & 1 & \frac{2 \gamma \Delta t}{\rho} \\
0 & 0 & 0 & 0 & 1 & -1 & 0
\end{array}\right] \\
& \boldsymbol{A}_{2}=\left[\begin{array}{ccccccc}
1-\frac{C V^{2}\left(\frac{1}{2}-\beta\right) \Delta t^{2}}{\rho} & \frac{C V^{2}\left(\frac{1}{2}-\beta\right) \Delta t^{2}}{\rho} & 0 & \Delta t & 0 & 0 & 0 \\
\frac{2 C V^{1}\left(\frac{1}{2}-\beta\right) \Delta t^{2}}{\rho} & 1-\frac{2 C V^{1}\left(\frac{1}{2}-\beta\right) \Delta t^{2}}{\rho} & 0 & 0 & \Delta t & 0 & \frac{2\left(\frac{1}{2}-\beta\right) \Delta t^{2}}{\rho} \\
0 & 0 & 1 & 0 & 0 & \Delta t & -\frac{2\left(\frac{1}{2}-\beta\right) \Delta t^{2}}{\rho} \\
-\frac{C V^{2}(1-\gamma) \Delta t}{\rho} & \frac{C V^{2}(1-\gamma) \Delta t}{\rho} & 0 & 1 & 0 & 0 & 0 \\
\frac{2 C V^{1}(1-\gamma) \Delta t}{\rho} & -\frac{2 C V^{1}(1-\gamma) \Delta t}{\rho} & 0 & 0 & 1 & 0 & \frac{2(1-\gamma) \Delta t}{\rho} \\
0 & 0 & 0 & 0 & 0 & 1 & -\frac{2(1-\gamma) \Delta t}{\rho} \\
0 & 0 & 0 & 0 & 0 & 0 & 0
\end{array}\right] \\
& \boldsymbol{c}_{0}=\left[\begin{array}{c}
\frac{\Delta t^{2}\left((1 / 2-\beta) b\left(t_{0}\right)+\beta b\left(t_{1}\right)\right)}{\rho} \\
-\frac{\Delta t^{2}\left((1 / 2-\beta) b\left(t_{0}\right)-\beta b\left(t_{1}\right)\right)}{\rho} \\
-\frac{\Delta t^{2}\left((1 / 2-\beta) b\left(t_{0}\right)-\beta b\left(t_{1}\right)\right)}{\rho} \\
\frac{\Delta t\left((1-\gamma) b\left(t_{0}\right)+\gamma b\left(t_{1}\right)\right)}{\rho} \\
-\frac{\Delta t\left((1-\gamma) b\left(t_{0}\right)-\gamma b\left(t_{1}\right)\right)}{\rho} \\
-\frac{\Delta t\left((1-\gamma) b\left(t_{0}\right)-\gamma b\left(t_{1}\right)\right)}{\rho} \\
0
\end{array}\right]
\end{aligned}
$$

The truncation results can now be obtained by solving (45) for $\boldsymbol{\tau}\left(t_{0}\right)$. After some simplification, the following local truncation error results, written 
in terms of the initial values, were obtained:

$$
\begin{aligned}
\tau_{u^{A, 1}}\left(t_{0}\right) & =\mathcal{O}\left(\Delta t^{2}\right) \\
\tau_{u^{A, 2}}\left(t_{0}\right) & =\mathcal{O}\left(\Delta t^{2}\right) \\
\tau_{u^{B, 2}}\left(t_{0}\right) & =\mathcal{O}\left(\Delta t^{2}\right) \\
\tau_{\dot{u}^{A, 1}}\left(t_{0}\right) & =-\frac{(2 \gamma-1) \Delta t\left(C V^{2}\left(\dot{u}^{2}\left(t_{0}\right)-\dot{u}^{1}\left(t_{0}\right)\right)+\dot{b}\left(t_{0}\right)\right)}{2 \rho}+\mathcal{O}\left(\Delta t^{2}\right) \\
\tau_{\dot{u}^{A, 2}}\left(t_{0}\right) & =\frac{(2 \gamma-1) \Delta t\left(C V^{1}\left(\dot{u}^{2}\left(t_{0}\right)-\dot{u}^{1}\left(t_{0}\right)\right)+\dot{b}\left(t_{0}\right)\right)}{2 \rho}+\mathcal{O}\left(\Delta t^{2}\right) \\
\tau_{\dot{u}^{B, 2}}\left(t_{0}\right) & =\frac{(2 \gamma-1) \Delta t\left(C V^{1}\left(\dot{u}^{2}\left(t_{0}\right)-\dot{u}^{1}\left(t_{0}\right)\right)+\dot{b}\left(t_{0}\right)\right)}{2 \rho}+\mathcal{O}\left(\Delta t^{2}\right) \\
\tau_{\lambda^{2}}\left(t_{0}\right) & =\mathcal{O}\left(\Delta t^{2}\right),
\end{aligned}
$$

where $\mathcal{O}\left(\Delta t^{2}\right)$ represents all terms of order $\Delta t^{2}$ and above.

It can be readily seen from the results above that the MTS method, with the simplifying assumptions made above, is second-order accurate if $\gamma=1 / 2$, and first-order accurate otherwise. This is the same condition as that of the undecomposed Newmark method applied to either a PD or classical problem. These results indicate that the MTS-PD approach will yield second-order accurate results under proper selection of the integration parameters.

Kinematic results for both the undecomposed system and the split system are plotted at both cells in Figures 7 and 8 . Those results correspond to Newmark- $\beta$ parameters of $\gamma=1 / 2$ and $\beta=1 / 4$. Comparisons between the analytical truncation errors (calculated via (50)) and exact numerical errors are also contained in the same plots. Log-log plots of the analytical and numerical errors for a range of time steps are also shown in Figures 9 and 10 to verify the claim of second-order accuracy. For these results, numerical errors refer to the difference between the exact solution and the solution of the split system. Results are shown for each cell and for two different choices of integration parameters $(\gamma=1 / 2, \beta=0$ and $\gamma=1 / 2, \beta=1 / 4)$. In 
all cases, the time step was taken to be $1 / 16 \mathrm{~s}$, each cell is separated by $1 \mathrm{~m}$, displacements are in meters, velocities are in meters per second, and accelerations are in meters per second squared.

Subdomain $A$, Cell 1
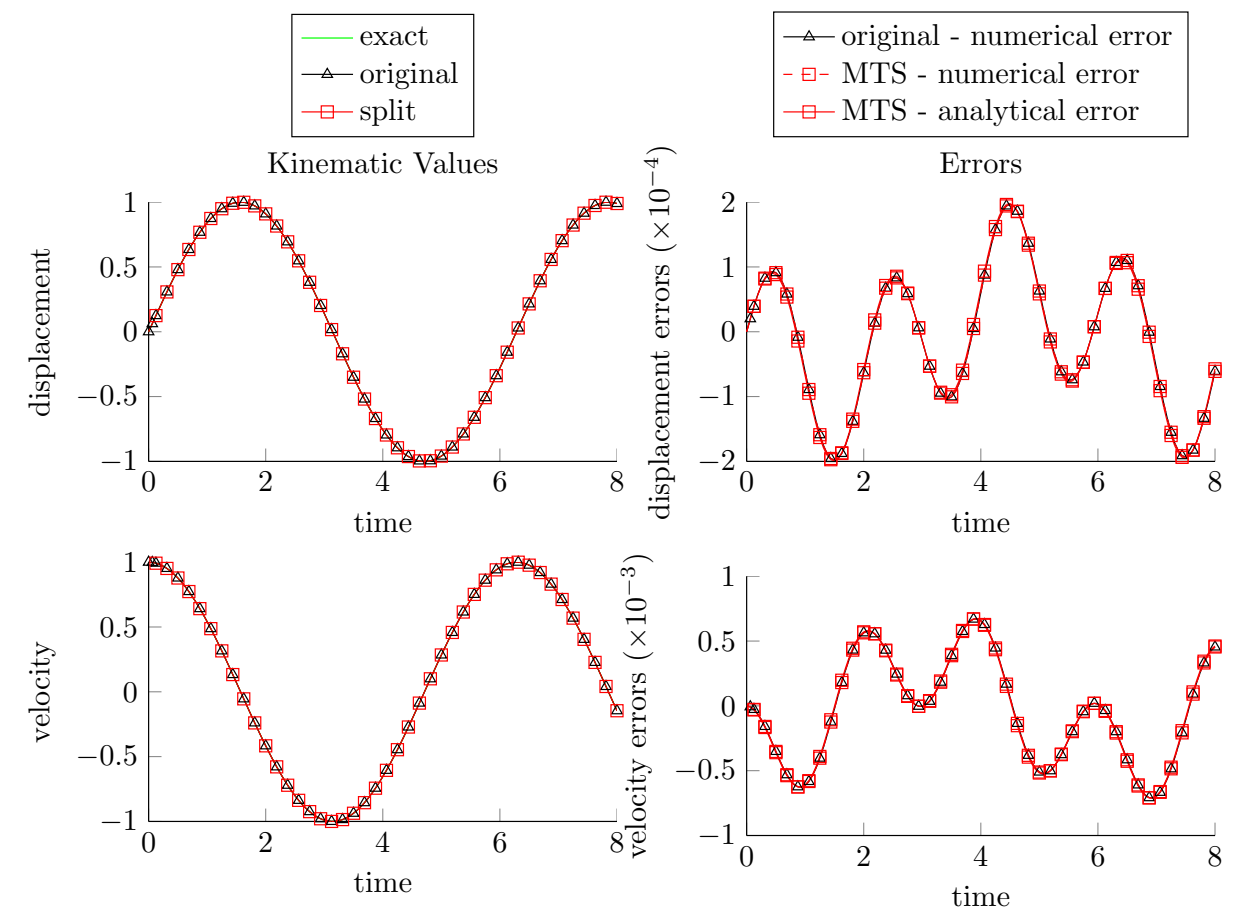

Figure 7: Kinematic values and errors for cell 1 of the model depicted in Figure 5

In addition to the cost advantages the MTS method offers, it inherits the accuracy of the PD spatial discretization and Newmark- $\beta$ temporal discretization it utilizes. This can be seen in Figures 7 and 8, where the (exact) errors for both the undecomposed PD method and the MTS method are very similar. These results show that the application of the MTS method does not appreciably degrade the accuracy of the underlying PD method. Figures 7 and 8 also show that the analytical error results, calculated using the local truncation errors, closely approximate the exact numerical errors. This result is further explored in Figures 9 and 10, where the second-order rate of 
Subdomain $A$ and $B$, Cell 2
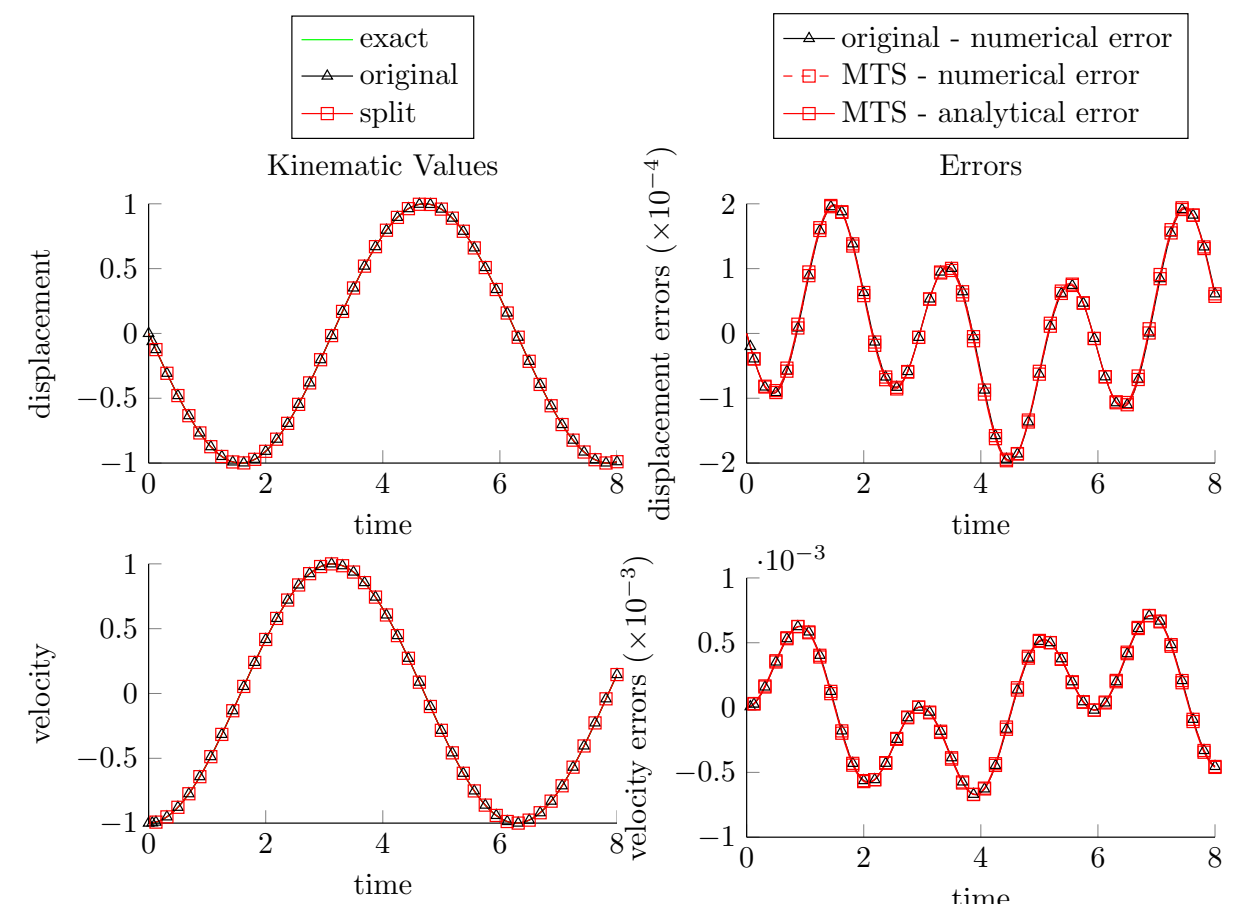

Figure 8: Kinematic values and errors for the cell 2 of the model depicted in Figure 5

Subdomain $A$, Cells $1 \& 2$

Subdomain $B$, Cell 2
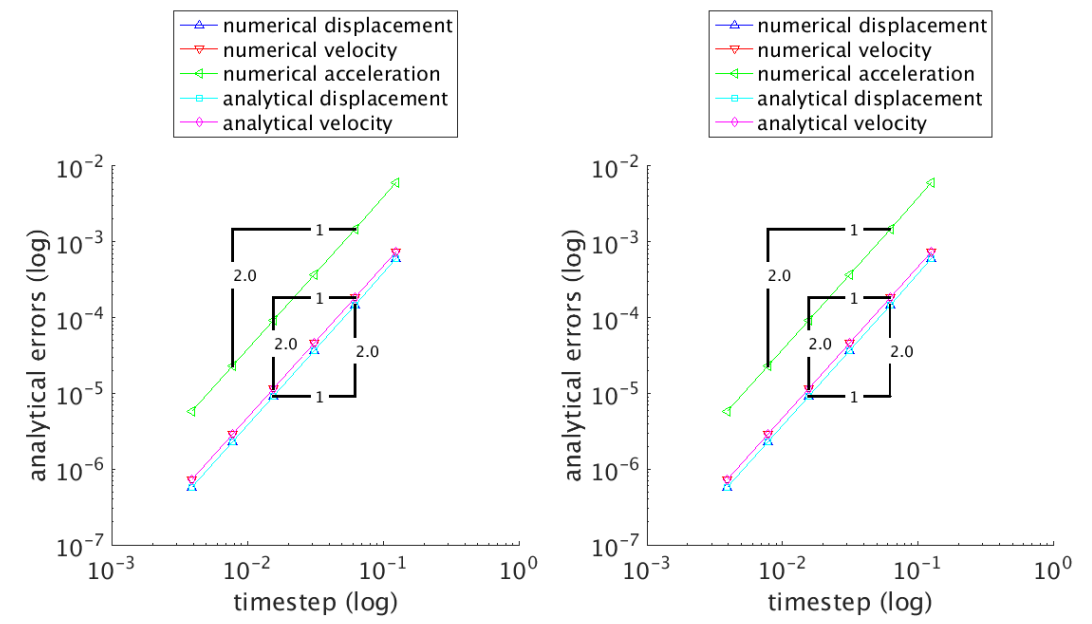

Figure 9: Errors with decreasing time step for $\beta=0$ 
Subdomain $A$, Cells $1 \& 2 \quad$ Subdomain $B$, Cell 2
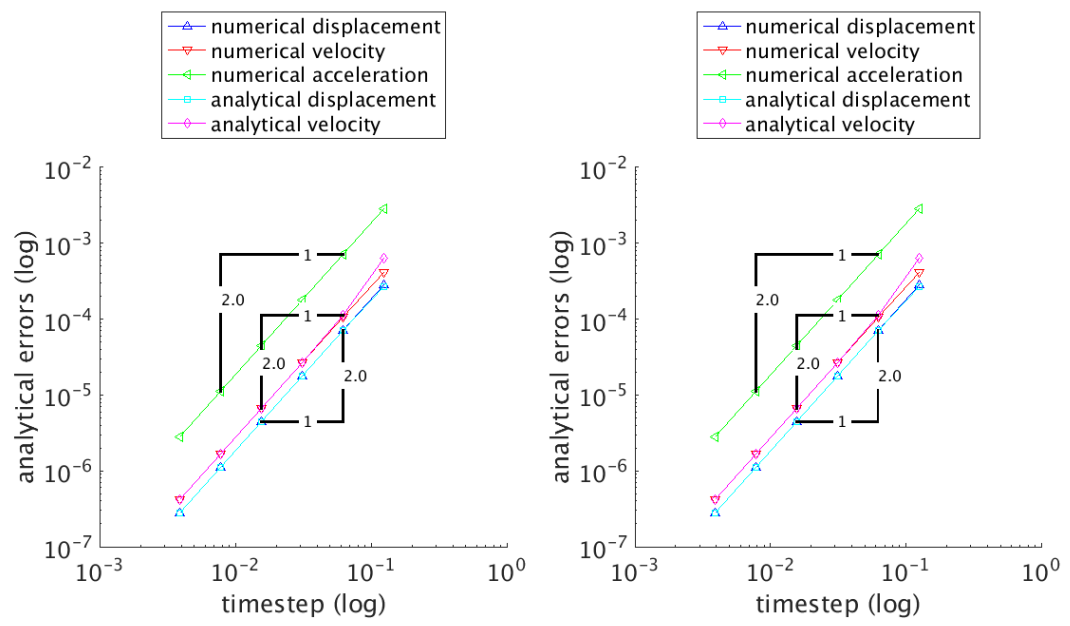

Figure 10: Errors with decreasing time step for $\beta=1 / 4$

convergence is verified both numerically and analytically. This reinforces the conclusion that the MTS method retains the second-order convergence of the PD method, and further confirms the validity of the convergence analysis. Additionally, Figures 9 and 10 demonstrate the robustness of the method and the validity of the error results for different integration methods. The secondorder convergence rate, with the restriction that $\gamma=1 / 2$, is seen not only in Newmark-based undecomposed classical problems and undecomposed PD, but has also been observed in classical MTS for arbitrarily large problems.

\section{Numerical Examples}

Two example problems are presented in this section. The first is the response of a one-dimensional bar loaded suddenly with tractions at each end. The second is a larger example showing fracture in a brittle plate under similar loading. A MATLAB prototype code, intended to demonstrate MTS$\mathrm{PD}$, was used for the numerical results. 


\subsection{One-Dimensional Peridynamic Bar Subject to End Forces}

A bar of length $20 \mathrm{~mm}$ was loaded suddenly with tractions on a region of width $\delta$ adjacent to the ends. This was accomplished by the application of a constant force density of $100 \times 10^{6} \mathrm{~N} / \mathrm{m}^{3}$ to simulate a step load, shown in Figure 11. Values of $\rho=2235 \mathrm{~kg} / \mathrm{m}^{3}$ and $E=72 \mathrm{GPa}$ were used for the density and Young's modulus, respectively.

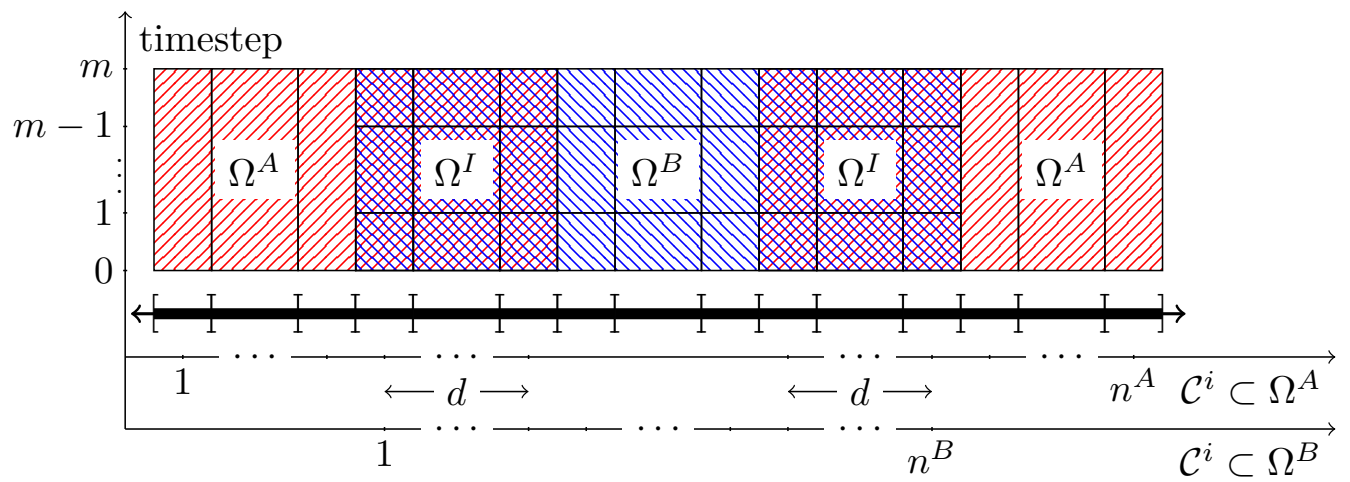

Figure 11: Space/time depiction of a one-dimensional bar loaded in tension

The problem was simulated with the undecomposed PD method and the MTS-PD approach for multiple different choices of the algorithmic parameters listed in Table 1. The bar was discretized with a default cell size of $\Delta x=2 \times 10^{-5} \mathrm{~m}$ i.e. $0.02 \mathrm{~mm}$ and integrated with a default time step of $1 \times 10^{-8} \mathrm{~s}$, with all cases using the same horizon $\delta=3 \times 10^{-4} \mathrm{~m}$ and being run for the same total time of $200 \mu \mathrm{s}$. Note: while the bar is a three-dimensional problem, it is discretized with a single cell along the width and height, and constrained to respond along the length, resulting in a "one-dimensional" peridynamic bar. For the MTS-PD approach, one subdomain $\left(\Omega^{B}\right)$ was taken to include the middle $10 \%$ of the total cells of the bar (shaded blue in Figure 11). The remainder of the domain $\left(\Omega^{A}\right.$, shaded red) was evaluated at a time step greater than or equal to the time step for subdomain $\Omega^{B}$, denoted with the time-step ratio $m$ in Table 1 . The two subdomains share an overlapping interface region $\Omega^{I}$ of width $d$ at each interface, such that 
$\delta=d \Delta x$.

Table 1: A table of the algorithm parameters and global errors for each case

\begin{tabular}{|c|c|c|c|c|c|c|c|c|}
\hline & case & $\beta^{A} \quad \beta^{B}$ & $\frac{\Delta t^{B}}{1 \times 10^{-8}}$ & $m$ & $\frac{\Delta x}{2 \times 10^{-5}}$ & $d_{e r r}(\%)$ & $v_{\text {err }}(\%)$ & $a_{e r r}(\%)$ \\
\hline \multirow{4}{*}{$\beta$} & 1 & $1 / 4 \quad 1 / 4$ & 1 & 2 & 1 & 0.19 & 1.79 & 9.80 \\
\hline & 2 & $1 / 4 \quad 0$ & 1 & 2 & 1 & 0.19 & 1.76 & 9.71 \\
\hline & 3 & $1 / 4 \quad 1 / 6$ & 1 & 2 & 1 & 0.19 & 1.78 & 9.77 \\
\hline & 4 & 0 & 1 & 2 & 1 & 0.11 & 1.12 & 7.50 \\
\hline \multirow{2}{*}{$m$} & $\overline{5}$ & $\begin{array}{lll}1 / 4 & 1 / 4\end{array}$ & $\overline{c 1}$ & 10 & 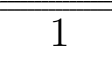 & $\bar{~} 1.03$ & 5.27 & 18.13 \\
\hline & 6 & $1 / 4 \quad 1 / 4$ & 1 & 1 & 1 & 0.07 & 0.92 & 6.77 \\
\hline \multirow{3}{*}{$\Delta t$} & 7 & $\begin{array}{ll}1 / 4 & 1 / 4\end{array}$ & $\overline{1 / 5}$ & 2 & $\overline{11}$ & 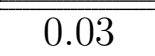 & $\overline{0.46}$ & 4.19 \\
\hline & 8 & $1 / 4 \quad 1 / 4$ & $1 / 5$ & 5 & 1 & 0.07 & 0.90 & 6.69 \\
\hline & 9 & $1 / 4 \quad 0$ & 2 & 5 & 1 & 1.03 & 5.27 & 18.12 \\
\hline \multirow{2}{*}{$\Delta x$} & 10 & $1 / 4 \quad 1 / 4$ & 1 & 2 & 5 & 0.28 & 2.45 & 11.59 \\
\hline & 11 & $1 / 4 \quad 1 / 4$ & 1 & 2 & $1 / 3$ & 0.04 & 0.42 & 2.33 \\
\hline \multirow{4}{*}{ 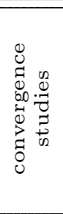 } & 12 & 0 & $1 / 10$ & 10 & 1 & 0.03 & 0.41 & 3.71 \\
\hline & 13 & 0 & 1 & 2 & $1 / 5$ & 0.11 & 1.16 & 7.64 \\
\hline & 14 & 0 & $1 / 5$ & 5 & $1 / 5$ & 0.04 & 0.57 & 5.09 \\
\hline & 15 & 0 & $1 / 10$ & 10 & $1 / 5$ & 0.04 & 0.57 & 5.09 \\
\hline \multirow{4}{*}{ 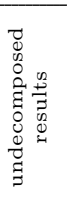 } & $\overline{16}$ & $\bar{\beta} \beta=1 / 4$ & 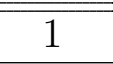 & ב-- & $\overline{1} 1$ & 0.07 & 0.93 & (6.81 \\
\hline & 17 & $\beta=0$ & 1 & -- & 1 & 0.03 & 0.44 & 3.91 \\
\hline & 18 & $\beta=0$ & $1 / 10$ & -- & $1 / 5$ & 0.00 & 0.05 & 0.62 \\
\hline & 19(ref.) & $\beta=0$ & $1 / 50$ & -- & $1 / 25$ & 0.00 & 0.00 & 0.00 \\
\hline
\end{tabular}

Sample results of displacement, velocity, acceleration and their errors for two specific cells, $\mathcal{C}^{A}$ and $\mathcal{C}^{I}$, are presented in Figure 12 for case 1 . All quantities are expressed in their SI units. Cell $\mathcal{C}^{A}$ is centered at the leftmost extremity of the bar $(x=0.01 \mathrm{~mm})$ within subdomain $\Omega^{A}$ and cell $\mathcal{C}^{I}$ is centered at $x=9.2 \mathrm{~mm}$ within the overlapping region $\Omega^{I}$. Note that the subplot for displacement in Figure 12 is presented with dual ordinate axes, the left axis for displacement of cell $\mathcal{C}^{A}$ and the right axis for displacement of cell $\mathcal{C}^{I}$. Instantaneous local errors, shown in Figure 12, are obtained using 
Table 2: A table of the timing results for each parameter case

\begin{tabular}{|c|c|c|c|c|}
\hline & case & Run-time (s) & $\begin{array}{c}\text { Run-time Per Time step } \Delta t^{A} \text { (ms) } \\
t:\left(t^{A}, t^{B}, t^{\lambda}\right)\end{array}$ & $\left(t^{B} / m, t^{\lambda} / m\right)$ \\
\hline \multirow{4}{*}{$\beta$} & $\overline{11}$ & 2.23 & $\bar{~} 1.24:(0.69,0.23,0.32)$ & $(0.11,0.16)$ \\
\hline & 2 & 1.95 & $1.08:(0.68,0.10,0.30)$ & $(0.05,0.15)$ \\
\hline & 3 & 2.20 & $1.22:(0.69,0.23,0.31)$ & $(0.11,0.16)$ \\
\hline & 4 & 0.86 & $0.48:(0.10,0.09,0.28)$ & $(0.05,0.14)$ \\
\hline \multirow{2}{*}{$m$} & 5 & 1.05 & $2.92:(0.77,1.11,1.04)$ & $(0.11,0.10)$ \\
\hline & 6 & 3.71 & $1.03:(0.68,0.13,0.23)$ & $(0.13,0.23)$ \\
\hline \multirow{3}{*}{$\Delta t$} & 7 & 10.86 & $1.21:(0.68,0.22,0.30)$ & $(0.11,0.15)$ \\
\hline & 8 & 7.67 & $2.13:(0.82,0.62,0.70)$ & $(0.12,0.14)$ \\
\hline & 9 & 0.59 & $1.63:(0.78,0.21,0.65)$ & $(0.04,0.13)$ \\
\hline \multirow{2}{*}{$\Delta x$} & 10 & 0.66 & $0.37:(0.08,0.10,0.18)$ & $(0.05,0.09)$ \\
\hline & 11 & 18.03 & $10.01:(7.62,1.51,0.88)$ & $(0.76,0.44)$ \\
\hline \multirow{4}{*}{ 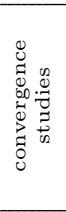 } & 12 & 5.83 & $1.62:(0.12,0.40,1.10)$ & $(0.04,0.11)$ \\
\hline & 13 & 6.40 & $3.55:(1.63,0.39,1.54)$ & $(0.19,0.77)$ \\
\hline & 14 & 15.39 & $4.28:(1.51,0.80,1.96)$ & $(0.16,0.39)$ \\
\hline & 15 & 21.77 & $6.05:(1.52,1.50,3.03)$ & $(0.15,0.30)$ \\
\hline \multirow{4}{*}{ 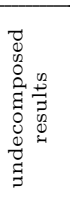 } & 16 & 2.47 & 0.69 & -- \\
\hline & 17 & 0.36 & 0.10 & -- \\
\hline & 18 & 40.16 & 1.12 & -- \\
\hline & 19(ref.) & 5268.10 & 29.27 & -- \\
\hline
\end{tabular}

case 19, an undecomposed PD model with a time step of $\Delta t=2 \times 10^{-10}$ s and cell spacing of $\Delta x=0.8 \mu \mathrm{m}$, as a reference solution. Instantaneous global errors, obtained by averaging local instantaneous errors for all the cells, are depicted in Figure 13. Average global errors, obtained by averaging the instantaneous global errors across all the time steps, are also provided in Table 1. One may note that, consistent with most finite difference schemes, the magnitude of errors grows from displacement to velocity to acceleration because of the underlying approximation of the time derivative. 

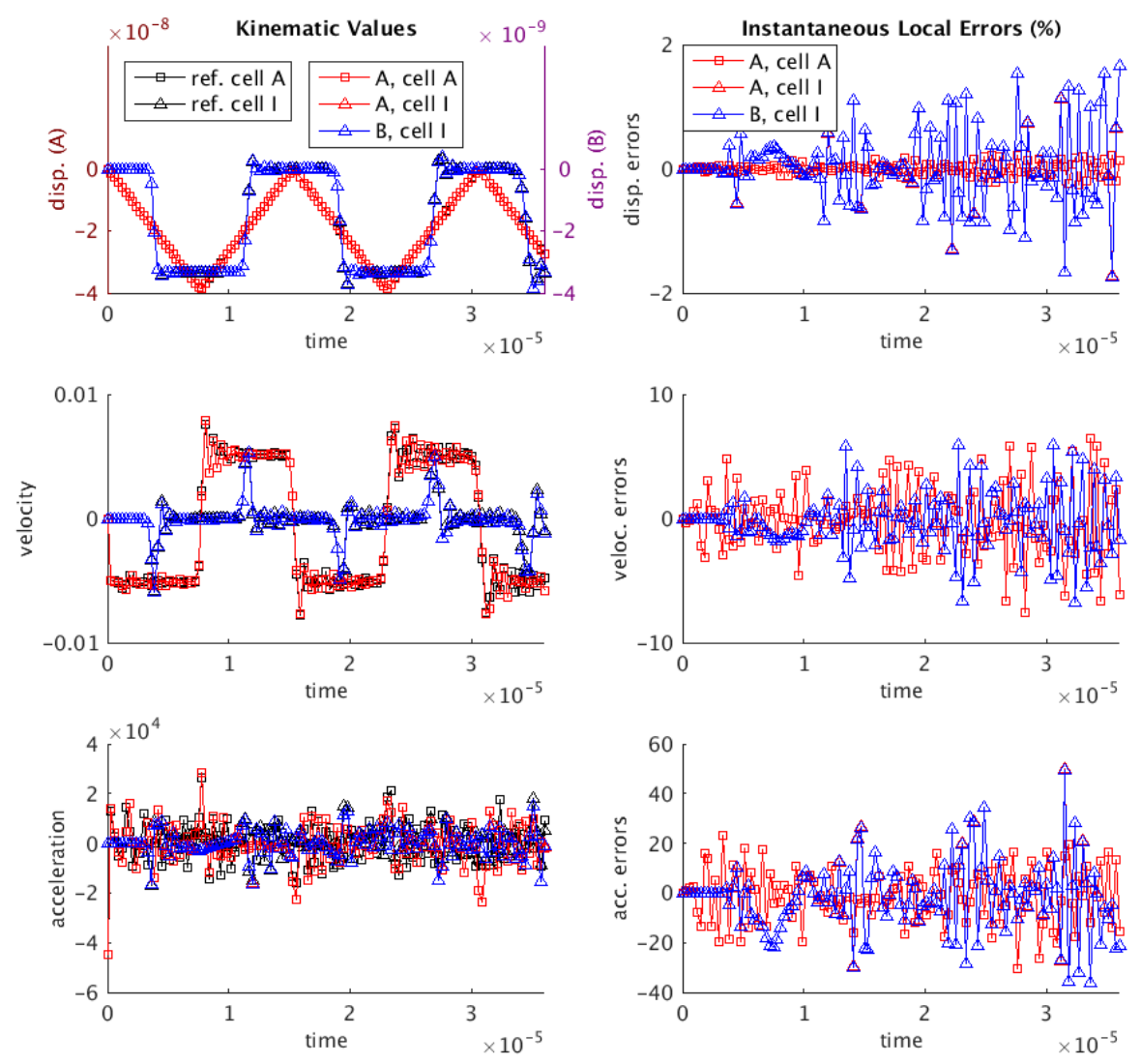

Figure 12: Kinematic and error results for case 1

A detailed break-up of the run-times for all the different cases listed in Table 1 are given in Table 2. The column $t:\left(t^{A}, t^{B}, t^{\lambda}\right)$ represents the run-time per time step. For MTS cases, this value was subdivided into the time to solve subdomain $A\left(t^{A}\right)$, the time to solve subdomain $B\left(t^{B}\right)$, and the time to solve for the interface forces $\boldsymbol{\lambda}$ and to update subdomains $A$ and $B\left(t^{\lambda}\right)$. One may note immediately that the solution time is heavily dependent on the size of the cell $\Delta x$. It can also be observed that for the same cell size, the time taken by implcit time integration $(\beta>0)$ is greater than that for explicit time integration $(\beta=0)$. For the MTS-PD cases, the 


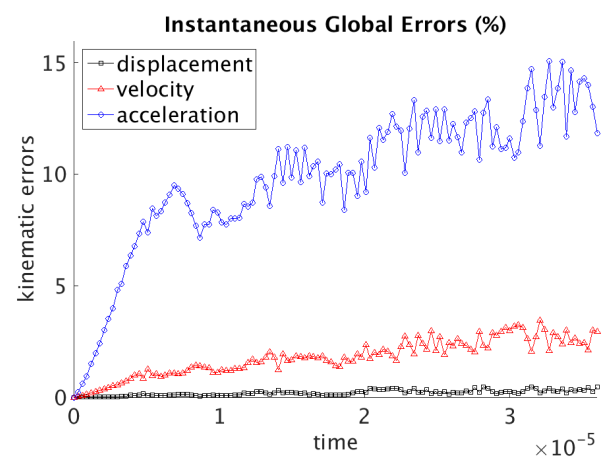

Figure 13: Global error results for case 1

times taken to solve subdomain $B\left(t^{B}\right)$ and the interface quantities $\left(t^{\lambda}\right)$ are proportional to the time-step ratio $m$. When normalized by $m, t^{B} / m$ and $t^{\lambda} / m$ approach a constant value for specific parametric choices, indicating that this component of the computational cost scales with time-step ratio linearly. For instance, for cases 1-9, when subdomain $B$ is chosen to be implicit $(\beta>0)$, the computational cost of solving subdomain $B$ per $\Delta t^{B}$ time step, is about $0.11 \mathrm{~s}$, while it is about 0.05 for cases where subdomain $B$ is solved explicitly $(\beta=0)$. A similar observation is also made in Prakash et al. [18]. This knowledge can help one partition a large PD problem domain into smaller subdomains and choose their time steps in a way that the computational load on a parallel machine is balanced evenly among the processors.

Tables 1 and 2 and Figures 12 and 13 indicate that the MTS-PD approach is viable for a wide range of time steps, time-step ratios between subdomains, spatial discretizations, problem sizes, and integration schemes. Cases 1-4 illustrate the robustness of the MTS-PD method with respect to the choice of time integration parameters and demonstrate that the results are not too sensitive to the choice of $\beta$. Cases 5 and 6 explore the effects of varying the time-step ratio. Comparing the computational costs, one may note that for case 5 the time taken to advance the solution by a $\Delta t^{A}=10 \times 10^{-8} \mathrm{~s}$ is $2.92 \mathrm{~ms}$, whereas the time taken to advance the solution for case 6 by the same amount 
would be $10 \times 1.03=10.3 \mathrm{~ms}$. This shows that the MTS-PD approach with large time-step ratios can be used to speed-up computations. However, for this simple 1-D problem, increasing the time-step ratio also leads to higher errors because the temporal characteristics of both subdomains $A$ and $B$ are the same and there is no physical reason to choose different time steps in these subdomains. As will be shown in the next section, for problems with different temporal characteristics, the MTS-PD approach can be used to speed-up the computation, without much loss of accuracy. Cases 7, 8, and 9 explore the effect of varying the time step. It is interesting to note that case 9 utilizes an implicit-explicit coupling with a time step for subdomain $A$ that is actually larger than the stability limit for the corresponding explicit method and still produces a stable solution. Cases 10 and 11 explore the effect of varying the cell size for the spatial discretization. Parameters in cases 12-15 were chosen to investigate the convergence of the MTS-PD method in space and time. Case 12 explores the effect of using a much smaller time step than the default case, case 13 considers a much finer spatial discretization, and cases 14 and 15 explore finer discretizations in both space and time. Taken together, these results highlight the robustness and accuracy of the MTS-PD method for solving a wide range of problems.

\subsection{Cracked Plate Example}

The following results correspond to a simulation of fracture in a $20 \times$ $10 \times 0.2 \mathrm{~mm}$ plate, discretized with cubic cells of volume $(0.2 \mathrm{~mm})^{3}$ and horizon $\delta=0.6 \mathrm{~mm}$. The plate was assumed to have the following properties: $\rho=2235 \mathrm{~kg} / \mathrm{m}^{3}, E=72 \mathrm{GPa}, G_{0}=204 \mathrm{~J} / \mathrm{m}^{2}$. These values were chosen to represent the properties of glass. The plate was loaded suddenly with tractions, represented by a constant force density of $20 \times 10^{6} \mathrm{~N} / \mathrm{m}^{3}$, along a region of width $\delta$ adjacent to the edges to simulate a step load (see Figure 2). An initial notch was introduced at the upper midpoint of the plate, with initial length equal to 1/4 of the total plate height, shaded yellow in Figure 2. This was included to facilitate cracking behavior in the plate. 
Results were obtained for both PD alone and also for the MTS-PD approach. For the MTS-PD approach, one subdomain $\left(\Omega^{B}\right)$ was taken to include $20 \%$ of the total cells surrounding the region initially containing the crack (shaded blue in Figure 2) and was evaluated at a time step of $\Delta t^{B}=1 \times 10^{-8} \mathrm{~s}$. The remainder of the domain (shaded red) was evaluated at a time step at least as large as $\Delta t^{B}$. Two different time steps, $1 \times 10^{-8}$ $\mathrm{s}$ and $10 \times 10^{-8} \mathrm{~s}$, were used for the undecomposed PD simulations. For all cases, the Newmark- $\beta$ integration parameters were taken as $\gamma=1 / 2, \beta=1 / 4$, and the displacement and position are given in meters.

Results showing the position and damage of all cells in the plate are shown in Figure 14 for several instants in time. The first is when the wave reaches the middle cracked portion (time $t_{A}$ ), the second is when the crack starts propagating (time $t_{B}$ ), and the third is when plate is cracked through (time $\left.t_{C}\right)$. Case 0 corresponds to the original undecomposed system evaluated at $\Delta t=1 \times 10^{-8} \mathrm{~s}$ and is used as the reference. Cases 1-3 are solved with a decomposed system with different MTS-PD parameters. Case 1 is solved with the same time step $\left(\Delta t^{A}=\Delta t^{B}=1 \times 10^{-8} \mathrm{~s}\right)$ for both subdomains (i.e., $m=1$ ). Case 2 is obtained with subdomain $A$ evaluated at a time step $m=2$ times larger than subdomain $B\left(\Delta t^{A}=2 \Delta t^{B}=2 \times 10^{-8} \mathrm{~s}\right)$ and Case 3 is evaluated similarly with $m=10$. Finally, Case 4 is computed with the undecomposed system again but using a time step of $\Delta t=10 \times 10^{-8} \mathrm{~s}$.

For the results in Figure 14, blue shades represent cells where the bonds are relatively unstretched from their initial configuration, while red shades represent cells with bonds that are experiencing a greater amount of stretch or completely broken. Cell coloration is based on the current state of the bonds connecting to each point, which is represented by a single value. For cells experiencing a net tensile loading, which is mainly what is seen in this simulation, this value ranges in magnitude from zero (initially unbroken) to one (completely isolated from the surrounding cells). Conversely, negative values correspond to cells in compression. This coloration value, $c_{n}$, is cal- 


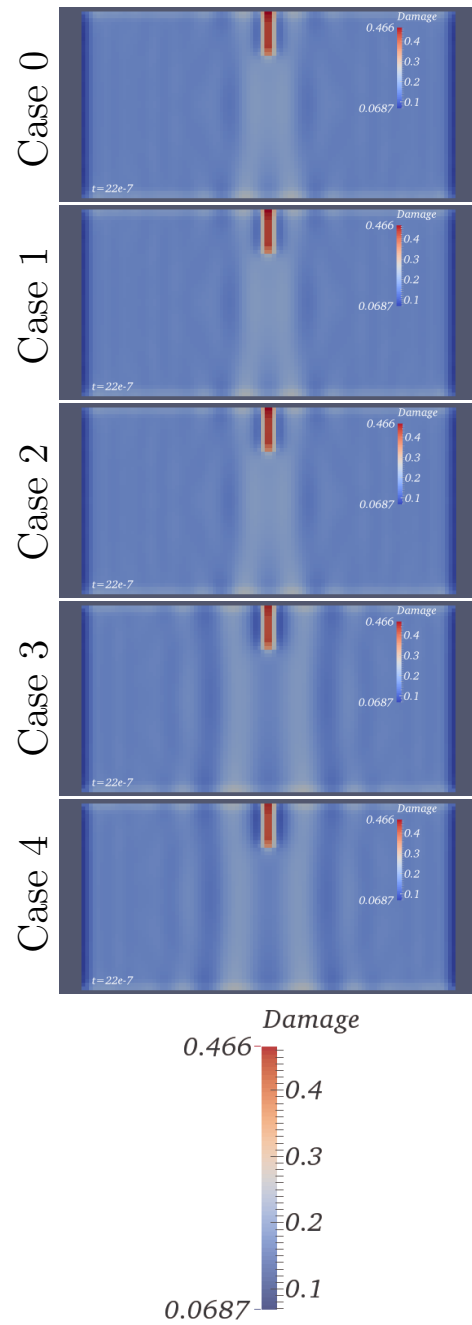

(a) time $t_{A}$
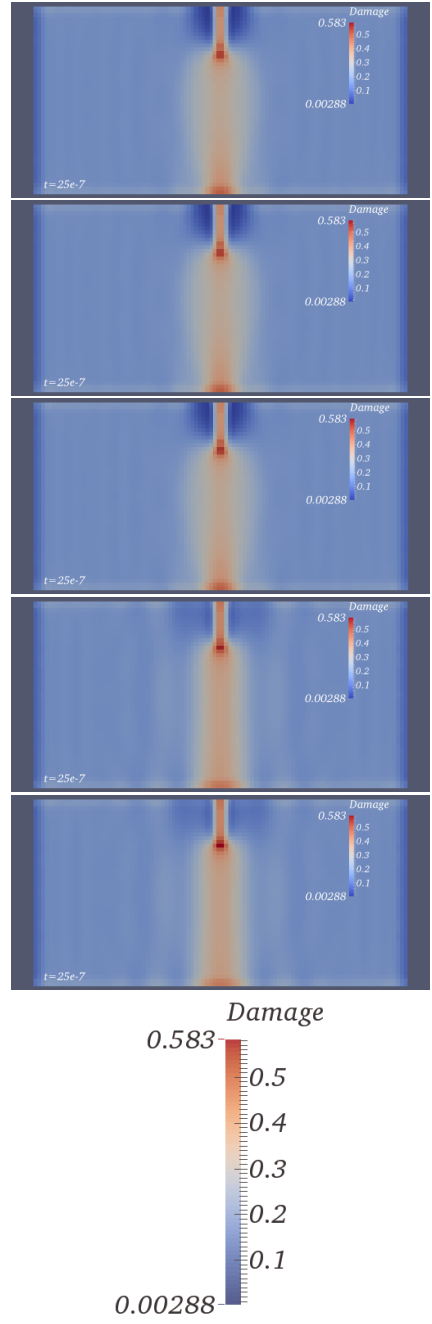

(b) time $t_{B}$
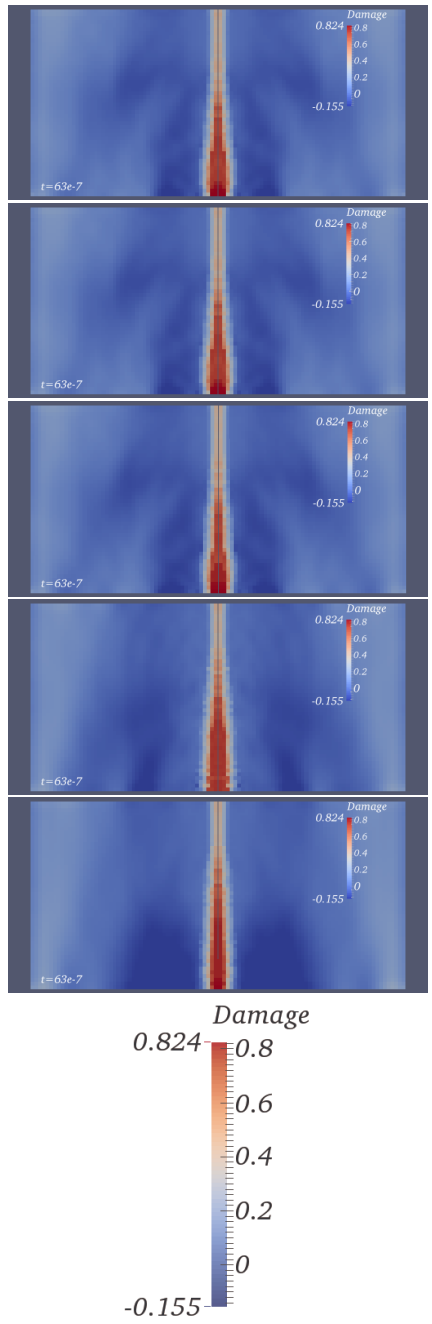

(c) time $t_{C}$

Figure 14: Results of the cracked-plate problem for MTS-PD at three different time-step ratios (cases 1-3) and undecomposed PD at two different time steps (cases 0 and 4)

culated for each cell $i$ using the relation

$$
c_{n}=\left(n_{b b}+\sum_{\mathcal{C}^{p} \subset \mathcal{H}_{x^{i}}} s^{p} / s_{0}\right) / n_{b}
$$

where $n_{b}$ is the number of initially unbroken bonds, $n_{b b}$ is the number of 
Table 3: The global errors of longitudinal displacements for each case of the cracked-plate problem

\begin{tabular}{|c|cccc|}
\hline case & description & through $t_{A}(\%)$ & through $t_{B}(\%)$ & through $t_{C}(\%)$ \\
\hline \multirow{4}{*}{1} & MTS-PD $, m=1, \Omega^{A}$ & 0 & 0 & 0 \\
\cline { 2 - 5 } & MTS-PD $, m=1, \Omega^{B}$ & 0 & 0 & 0 \\
\cline { 2 - 5 } & MTS-PD $, m=1, \Omega$ & 0 & 0 & 0 \\
\hline \multirow{4}{*}{2} & MTS-PD, $m=2, \Omega^{A}$ & $1.1 \times 10^{-5}$ & $1.0 \times 10^{-5}$ & $8.4 \times 10^{-4}$ \\
\cline { 2 - 5 } & MTS-PD, $m=2, \Omega^{B}$ & $2.2 \times 10^{-6}$ & $2.7 \times 10^{-6}$ & $1.1 \times 10^{-3}$ \\
\cline { 2 - 5 } & MTS-PD, $m=2, \Omega$ & $9.2 \times 10^{-6}$ & $8.9 \times 10^{-6}$ & $8.9 \times 10^{-4}$ \\
\hline \multirow{4}{*}{3} & MTS-PD, $m=10, \Omega^{A}$ & $5.2 \times 10^{-5}$ & $5.3 \times 10^{-5}$ & $8.4 \times 10^{-3}$ \\
\cline { 2 - 5 } & MTS-PD, $m=10, \Omega^{B}$ & $3.4 \times 10^{-5}$ & $6.4 \times 10^{-5}$ & $9.3 \times 10^{-3}$ \\
\cline { 2 - 5 } & MTS-PD $, m=10, \Omega$ & $4.9 \times 10^{-5}$ & $5.5 \times 10^{-5}$ & $8.6 \times 10^{-3}$ \\
\hline \multirow{4}{*}{4} & $\mathrm{PD}, \Delta t=10 \times 10^{-8} \mathrm{~s}, \Omega^{A}$ & $5.3 \times 10^{-5}$ & $5.4 \times 10^{-5}$ & $1.4 \times 10^{-2}$ \\
\cline { 2 - 5 } & $\mathrm{PD}, \Delta t=10 \times 10^{-8} \mathrm{~s}, \Omega^{B}$ & $3.4 \times 10^{-5}$ & $5.9 \times 10^{-5}$ & $1.6 \times 10^{-2}$ \\
\cline { 2 - 5 } & $\mathrm{PD}, \Delta t=10 \times 10^{-8} \mathrm{~s}, \Omega$ & $5.1 \times 10^{-5}$ & $5.6 \times 10^{-5}$ & $1.5 \times 10^{-2}$ \\
\hline
\end{tabular}

currently broken bonds, and $s^{p}$ is the current stretch of a cell $p$ in the horizon of cell $i$. In this way, $c_{n}$ can be thought of as the "closeness to being broken" of a given cell.

The average errors relative to the undecomposed $\mathrm{PD}$ solution at $\Delta t=$ $1 \times 10^{-8} \mathrm{~s}$ are summarized in Table 3 . These values were obtained by averaging instantaneous local errors in both space and time. It can be seen that the accuracy of the undecomposed PD solution with a time step of $10 \times 10^{-8}$ $\mathrm{s}$ is lower than any of the MTS-PD results. An interesting comparison can be made by comparing this undecomposed PD solution to the results for a time-step ratio of $m=10$, where the MTS method achieves a more accurate solution while still keeping computational costs low. The MTS-PD decomposition with $m=1$ (case 1 ) results are equal within numerical precision to the PD results at the same time step, which is expected. MTS is generally utilized to attain higher accuracy in regions where it is needed most. However, one exciting feature of the MTS method observed in these results is the 
reduction of errors in all regions. This can be seen in Table 3 at time $t_{C}$ when comparing the results of the undecomposed PD solution with $\Delta t=10 \times 10^{-8}$ s (case 4 ) and the MTS method with $m=10$ (case 3 ). The errors in $\Omega^{B}$ have become significantly lower than the corresponding region in the undecomposed PD simulation. This has the cumulative effect of lowering errors in $\Omega^{A}$, which is integrated using the same time step is used for both methods. The computational saving that MTS offers was also observed for these results: a 25\% speed-up over undecomposed PD (case 0) was recorded for the MTS-PD approach with $m=2$, and MTS-PD with $m=10$ ran 2.5 times faster.

These results reinforce the conclusions drawn from the 1D bar problem. The MTS-PD approach provides significant computational gains for a range of problem sizes and time-step ratios without significantly affecting accuracy. Indeed, the cost analysis in section 5.1 has shown that the MTS approach offers increasing opportunities for computational gain for larger problem sizes.

\section{Conclusions}

The multi-time-step peridynamic (MTS-PD) approach enables a novel development for peridynamic theory - the ability to relax temporal restrictions as needed. In the present work, the stability, accuracy and computational costs associated with this approach have been studied, analytically and numerically. It is demonstrated that the MTS-PD approach affords several advantages over conventional peridynamic methods where one is constrained to solve the entire problem with a uniform time step. Decomposing a large peridynamic problem domain into two or more subdomains and allowing different subdomains to use different time steps leads to greatly reduced computational costs while having a minimal impact on solution accuracy.

Theoretical arguments and numerical results show that the MTS-PD approach is stable as long as the component subdomain computations are stable i.e the process of coupling different subdomains does not lead to instability. 
The consistency and accuracy of this approach were studied using a detailed error analysis in the context of a small test problem and it was found that the MTS-PD approach preserves the convergence rates of the component subdomain solutions and of the global problem solution. Several different choices of MTS-PD problem parameters were simulated numerically using two test problems, wave propagation in a one-dimensional bar and crack propagation in a two-dimensional plate. The results show that the MTS-PD approach, when used judiciously, reduces the computational cost without having a significant impact on the accuracy of a refined undecomposed PD model for a wide range of integration parameters, time steps, spatial discretizations, and problem sizes.

\section{Acknowledgments}

This research is supported by the Department of Energy under contract DE-FC02-12ER26104.

\section{References}

[1] B. Karihaloo, Q. Xiao, Modelling of stationary and growing cracks in FE framework without remeshing: a state-of-the-art review, Computers \& Structures 81 (3) (2003) 119-129, doi:10.1016/S0045-7949(02)00431-5.

[2] Y. Abdelaziz, A. Hamouine, A survey of the extended finite element, Computers \& Structures 86 (11) (2008) 1141-1151, doi:10.1016/j.compstruc.2007.11.001.

[3] T. Belytschko, T. Black, Elastic Crack Growth in Finite Elements with Minimal Remeshing, International Journal for Numerical Methods in Engineering 45 (5) (1999) 601-620, doi:10.1002/(SICI)10970207(19990620)45:5<601::AID-NME598>3.0.CO;2-S. 
[4] T. Strouboulis, I. Babuška, K. Copps, The design and analysis of the Generalized Finite Element Method, Computer Methods in Applied Mechanics and Engineering 181 (1) (2000) 43-69, doi:10.1016/S00457825(99)00072-9.

[5] C. A. Duarte, J. T. Oden, An h-p adaptive method using clouds, Computer Methods in Applied Mechanics and Engineering 139 (1) (1996) 237-262, doi:10.1016/S0045-7825(96)01085-7.

[6] J. M. Melenk, I. Babuška, The partition of unity finite element method: Basic theory and applications, Computer Methods in Applied Mechanics and Engineering 139 (1) (1996) 289-314, doi:10.1016/S00457825(96)01087-0.

[7] M. Elices, G. Guinea, J. Gomez, J. Planas, The cohesive zone model: advantages, limitations and challenges, Engineering Fracture Mechanics 69 (2) (2002) 137-163, doi:10.1016/S0013-7944(01)00083-2.

[8] T. Belytschko, Y. Y. Lu, L. Gu, Element-free Galerkin methods, International Journal for Numerical Methods in Engineering 37 (2) (1994) 229-256, doi:10.1002/nme.1620370205.

[9] M. Liu, G. Liu, Smoothed particle hydrodynamics (SPH): an overview and recent developments, Archives of Computational Methods in Engineering 17 (1) (2010) 25-76.

[10] W. K. Liu, S. Jun, S. Li, J. Adee, T. Belytschko, Reproducing kernel particle methods for structural dynamics, International Journal for Numerical Methods in Engineering 38 (1995) 1655-1679.

[11] E. Kröner, Elasticity theory of materials with long range cohesive forces, International Journal of Solids and Structures 3 (5) (1967) 731-742, doi:10.1016/0020-7683(67)90049-2. 
[12] I. A. Kunin, Elastic media with microstructure. I-One-dimensional models, vol. 26, Springer-Verlag, doi:10.1007/978-3-642-81748-9, 1982.

[13] I. A. Kunin, Elastic Media with Microstructure II: Three-Dimensional Models, vol. 2, Springer-Verlag, doi:10.1007/978-3-642-81960-5, 1983.

[14] D. Rogula, Introduction to Nonlocal Theory of Material Media, in: Nonlocal Theory of Material Media, Springer, 123-222, doi:10.1007/978-37091-2890-9_3, 1982.

[15] S. A. Silling, Reformulation of elasticity theory for discontinuities and long-range forces, Journal of the Mechanics and Physics of Solids 48 (1) (2000) 175-209, doi:10.1016/S0022-5096(99)00029-0.

[16] S. A. Silling, M. Epton, O. Weckner, J. Xu, E. Askari, Peridynamic States and Constitutive Modeling, Journal of Elasticity 88 (2) (2007) 151-184, doi:10.1007/s10659-007-9125-1.

[17] A. Prakash, K. Hjelmstad, A FETI-based multi-time-step coupling method for Newmark schemes in structural dynamics, International Journal for Numerical Methods in Engineering 61 (13) (2004) 21832204, doi:10.1002/nme.1136.

[18] A. Prakash, E. Taciroglu, K. D. Hjelmstad, Computationally efficient multi-time-step method for partitioned time integration of highly nonlinear structural dynamics, Computers \& Structures 133 (2014) 51-63, doi:10.1016/j.compstruc.2013.11.013.

[19] A. Prakash, Multi-Time-Step Domain Decomposition and Coupling Methods for Non-linear Structural Dynamics, Ph.D. thesis, University of Illinois at Urbana-Champaign, URL https://engineering. purdue . edu/ aprakas/PrakashDissertation.pdf, 2007. 
[20] S. Silling, R. Lehoucq, Peridynamic Theory of Solid Mechanics, Advances in Applied Mechanics 44 (1) (2010) 73-166, doi:10.1016/S00652156(10)44002-8.

[21] E. Askari, F. Bobaru, R. Lehoucq, M. Parks, S. Silling, O. Weckner, Peridynamics for multiscale materials modeling, in: Journal of Physics: Conference Series, vol. 125, IOP Publishing, 012078, doi:10.1088/17426596/125/1/012078, 2008.

[22] S. A. Silling, E. Askari, Peridynamic Modeling of Impact Damage, in: ASME/JSME 2004 Pressure Vessels and Piping Conference, American Society of Mechanical Engineers, 197-205, doi:10.1115/PVP2004-3049, 2004.

[23] F. Bobaru, Influence of van der Waals forces on increasing the strength and toughness in dynamic fracture of nanofibre networks: a peridynamic approach, Modelling and Simulation in Materials Science and Engineering 15 (5) (2007) 397, doi:10.1088/0965-0393/15/5/002.

[24] F. Bobaru, S. A. Silling, Peridynamic 3D models of nanofiber networks and carbon nanotube-reinforced composites, in: Materials Processing and Design: Modeling, Simulation and Applications-NUMIFORM 2004Proceedings of the 8th International Conference on Numerical Methods in Industrial Forming Processes, vol. 712, AIP Publishing, 1565-1570, doi:10.1063/1.1766752, 2004.

[25] P. Seleson, M. L. Parks, M. Gunzburger, Peridynamic state-based models and the embedded-atom model, Communications in Computational Physics 15 (1) (2014) 179-205.

[26] P. Seleson, M. L. Parks, M. Gunzburger, R. B. Lehoucq, Peridynamics as an upscaling of molecular dynamics, Multiscale Modeling \& Simulation 8 (1) (2009) 204-227. 
[27] W. Gerstle, N. Sau, S. Silling, Peridynamic modeling of concrete structures, Nuclear Engineering and Design 237 (12) (2007) 1250-1258, doi:10.1016/j.nucengdes.2006.10.002.

[28] S. Silling, E. Askari, A meshfree method based on the peridynamic model of solid mechanics, Computers \& structures 83 (17) (2005) 1526-1535, doi:10.1016/j.compstruc.2004.11.026.

[29] S. A. Silling, M. Zimmermann, R. Abeyaratne, Deformation of a peridynamic bar, Journal of Elasticity 73 (1-3) (2003) 173-190, doi:10.1023/B:ELAS.0000029931.03844.4f.

[30] P. Seleson, Improved one-point quadrature algorithms for twodimensional peridynamic models based on analytical calculations, Computer Methods in Applied Mechanics and Engineering 282 (2014) 184217.

[31] N. M. Newmark, A Method of Computation for Structural Dynamics, in: Proc. ASCE, vol. 85, 67-94, 1959.

[32] W. C. Swope, H. C. Andersen, P. H. Berens, K. R. Wilson, A computer simulation method for the calculation of equilibrium constants for the formation of physical clusters of molecules: Application to small water clusters, The Journal of Chemical Physics 76 (1) (1982) 637-649.

[33] C. A. Felippa, K. Park, C. Farhat, Partitioned analysis of coupled mechanical systems, Computer Methods in Applied Mechanics and Engineering 190 (24) (2001) 3247-3270, doi:10.1016/S0045-7825(00)00391-1.

[34] C. Felippa, K. Park, Staggered transient analysis procedures for coupled mechanical systems: Formulation, Computer Methods in Applied Mechanics and Engineering 24 (1) (1980) 61-111, doi:10.1016/00457825(80)90040-7. 
[35] C. Farhat, K. G. Van der Zee, P. Geuzaine, Provably second-order timeaccurate loosely-coupled solution algorithms for transient nonlinear computational aeroelasticity, Computer Methods in Applied Mechanics and Engineering 195 (17) (2006) 1973-2001, doi:10.1016/j.cma.2004.11.031.

[36] T. Belytschko, H. Yen, R. Mullen, Mixed methods for time integration, Computer Methods in Applied Mechanics and Engineering 17 (1979) 259-275, doi:10.1016/0045-7825(79)90022-7.

[37] T. Belytschko, R. Mullen, Stability of explicit-implicit mesh partitions in time integration, International Journal for Numerical Methods in Engineering 12 (10) (1978) 1575-1586, doi:10.1002/nme.1620121008.

[38] W. Liu, T. Belytschko, Mixed-time implicit-explicit finite elements for transient analysis, Computers \& Structures 15 (4) (1982) 445-450, doi:10.1016/0045-7949(82)90079-7.

[39] M. Neal, T. Belytschko, Explicit-explicit subcycling with non-integer time step ratios for structural dynamic systems, Computers \& Structures 31 (6) (1989) 871-880, doi:10.1016/0045-7949(89)90272-1.

[40] T. J. R. Hughes, W. Liu, Implicit-Explicit Finite Elements in Transient Analysis: Stability Theory, Journal of Applied Mechanics, ASME 45 (1978) 371-374, transactions of the ASME, doi:10.1115/1.3424304.

[41] T. J. R. Hughes, W. Liu, Implicit-Explicit Finite Elements in Transient Analysis: Implementation and Numerical Examples, Journal of Applied Mechanics, ASME 45 (1978) 375-378, transactions of the ASME, doi:10.1115/1.3424305.

[42] P. Smolinski, An explicit multi-time step integration method for second order equations, Computer Methods in Applied Mechanics and Engineering 94 (1992) 25-34, doi:10.1016/0045-7825(92)90155-D. 
[43] P. Smolinski, Stability analysis of a multi-time step explicit integration method, Computer Methods in Applied Mechanics and Engineering 95 (3) (1992) 291-300, doi:10.1016/0045-7825(92)90188-P.

[44] W. Daniel, The subcycled Newmark algorithm, Computational Mechanics 20 (3) (1997) 272-281, doi:10.1007/s004660050248.

[45] W. Daniel, A study of the stability of subcycling algorithms in structural dynamics, Computer Methods in Applied Mechanics and Engineering 156 (1) (1998) 1-13, doi:10.1016/S0045-7825(97)00140-0.

[46] W. Daniel, A partial velocity approach to subcycling structural dynamics, Computer Methods in Applied Mechanics and Engineering 192 (3) (2003) 375-394, doi:10.1016/S0045-7825(02)00518-2.

[47] E. Sotelino, A concurrent explicit-implicit algorithm in structural dynamics, Computers \& Structures 51 (2) (1994) 181-190, doi:10.1016/0045-7949(94)90048-5.

[48] C. Farhat, L. Crivelli, F. X. Roux, Transient FETI methodology for large-scale parallel implicit computations in structural mechanics, International Journal for Numerical Methods in Engineering 37 (1994) 1945-1975, doi:10.1002/nme.1620371111.

[49] C. Farhat, P. Chen, J. Mandel, A scalable Lagrange multiplier based domain decomposition method for time-dependent problems, International Journal for Numerical Methods in Engineering 38 (22) (1995) 3831-3853, doi:10.1002/nme.1620382207.

[50] A. Gravouil, A. Combescure, Multi-time-step explicit-implicit method for non-linear structural dynamics, International Journal for Numerical Methods in Engineering 50 (1) (2001) 199-225, doi:10.1002/10970207(20010110)50:1<199::AID-NME132>3.0.CO;2-A. 
[51] A. Combescure, A. Gravouil, A numerical scheme to couple subdomains with different time-steps for predominantly linear transient analysis, Computer Methods in Applied Mechanics and Engineering 191 (2002) 1129-1157, doi:10.1016/S0045-7825(01)00190-6.

[52] P. Guidault, O. Allix, L. Champaney, C. Cornuault, A multiscale extended finite element method for crack propagation, Computer Methods in Applied Mechanics and Engineering 197 (5) (2008) 381-399, doi:10.1016/j.cma.2007.07.023.

[53] P. Guidault, O. Allix, L. Champaney, J. Navarro, A two-scale approach with homogenization for the computation of cracked structures, Computers \& Structures 85 (17) (2007) 1360-1371, doi:10.1016/j.compstruc.2006.08.085.

[54] E. Wyart, M. Duflot, D. Coulon, P. Martiny, T. Pardoen, J. Remacle, F. Lani, Substructuring FE-XFE approaches applied to threedimensional crack propagation, Journal of Computational and Applied Mathematics 215 (2) (2008) 626-638, doi:10.1016/j.cam.2006.03.066.

[55] E. Wyart, D. Coulon, M. Duflot, T. Pardoen, J. Remacle, F. Lani, A substructured FE-shell/XFE-3D method for crack analysis in thinwalled structures, International Journal for Numerical Methods in Engineering 72 (7) (2007) 757-779, doi:10.1002/nme.2029.

[56] S. Xiao, T. Belytschko, A bridging domain method for coupling continua with molecular dynamics, Computer Methods in Applied Mechanics and Engineering 193 (17) (2004) 1645-1669, doi:10.1016/j.cma.2003.12.053.

[57] H. Rafii-Tabar, L. Hua, M. Cross, A multi-scale atomistic-continuum modelling of crack propagation in a two-dimensional macroscopic plate, Journal of Physics: Condensed Matter 10 (11) (1998) 2375-2387, doi:10.1088/0953-8984/10/11/003. 
[58] O. Lloberas-Valls, D. Rixen, A. Simone, L. Sluys, Domain decomposition techniques for the efficient modeling of brittle heterogeneous materials, Computer Methods in Applied Mechanics and Engineering 200 (13) (2011) 1577-1590, doi:10.1016/j.cma.2011.01.008.

[59] T. Belytschko, D. Organ, Y. Krongauz, A coupled finite elementelement-free Galerkin method, Computational Mechanics 17 (3) (1995) 186-195, doi:10.1007/BF00364080.

[60] B. Aksoylu, M. L. Parks, Variational theory and domain decomposition for nonlocal problems, Applied Mathematics and Computation 217 (14) (2011) 6498-6515, doi:10.1016/j.amc.2011.01.027.

[61] P. Seleson, M. Gunzburger, M. L. Parks, Interface problems in nonlocal diffusion and sharp transitions between local and nonlocal domains, Computer Methods in Applied Mechanics and Engineering 266 (2013) 185-204. 